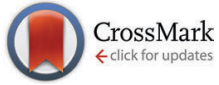

Cite this: J. Mater. Chem. C, 2016 , 4, 9823

Received 6th September 2016, Accepted 29th September 2016

DOI: $10.1039 / \mathrm{c} 6 \mathrm{tc0} 03874 \mathrm{c}$

www.rsc.org/MaterialsC

\section{Modular synthesis of simple cycloruthenated complexes with state-of-the-art performance in p-type DSCs $\dagger$}

\author{
Felix Brunner, ${ }^{a}$ Nathalie Marinakis, ${ }^{a}$ Cedric Wobill, ${ }^{a}$ Markus Willgert, ${ }^{a}$ Cathrin D. Ertl, ${ }^{a}$ \\ Tatjana Kosmalski, ${ }^{a}$ Markus Neuburger, ${ }^{a}$ Biljana Bozic-Weber, ${ }^{a}$ Thilo Glatzel, ${ }^{b}$ \\ Edwin C. Constable ${ }^{a}$ and Catherine E. Housecroft ${ }^{\star^{a}}$
}

\begin{abstract}
A modular approach based on Suzuki-Miyaura cross coupling and Miyaura borylation has been used to prepare two cyclometallated $\left[\mathrm{Ru}\left(\mathrm{N}^{\wedge} \mathrm{N}\right)_{2}\left(\mathrm{C}^{\wedge} \mathrm{N}\right)\right]^{+}$complexes which possess either a carboxylic or phosphonic acid group attached via a phenylene spacer to the 4-position of the pyridine ring in the $\mathrm{C}^{\wedge} \mathrm{N}$ ligand. The key intermediate in the synthetic pathway is $\left[\mathrm{Ru}(\mathrm{bpy})_{2}(\mathbf{1})\right]^{+}$where bpy $=2,2^{\prime}$-bipyridine and $\mathrm{H} \mathbf{1}$ is 4 -chloro-2phenylpyridine. The crystal structure of $\left[R u(b p y)_{2}(\mathbf{1})\right]\left[P F_{6}\right]$ is presented. Reaction of $\left[R u(b p y)_{2}(\mathbf{1})\right]\left[P F_{6}\right]$ with 4-carboxyphenylboronic acid leads to $\left[\mathrm{Ru}(\mathrm{bpy})_{2}\left(\mathrm{H}_{6}\right)\right]\left[\mathrm{PF}_{6}\right]$, while the phosphonic acid analogue is isolated as the zwitterion $\left[\mathrm{Ru}(\mathrm{bpy})_{2}(\mathrm{H} 5)\right]$. The cyclometallated complexes have been characterized by mass spectrometry, multinuclear NMR spectroscopy, absorption spectroscopy and electrochemistry. $\left[R u(b p y)_{2}(\mathbf{5})\right]$ adsorbs onto $\mathrm{NiO} \mathrm{FTO} / \mathrm{NiO}$ electrodes (confirmed by solid-state absorption spectroscopy) and its performance in p-type dye-sensitized solar cells (DSCs) has been compared to that of the standard dye P1; two-screen printed layers of $\mathrm{NiO}$ give better DSC performances than one layer. Duplicate DSCs containing $\left[\mathrm{Ru}(\mathrm{bpy})_{2}(\mathrm{H} 5)\right]$ achieve short-circuit current densities $\left(J_{\mathrm{SC}}\right)$ of 3.38 and $3.34 \mathrm{~mA} \mathrm{~cm}{ }^{-2}$ and photoconversion efficiencies $(\eta)$ of 0.116 and $0.109 \%$, respectively, compared to values of $J_{\mathrm{SC}}=1.84$ and $1.96 \mathrm{~mA} \mathrm{~cm}{ }^{-2}$ and $\eta=0.057$ and $0.051 \%$ for P1. Despite its simple dye structure, the performance of $\left[\mathrm{Ru}(\mathrm{bpy})_{2}(\mathrm{H} 5)\right]$ parallels the best-performing cyclometallated ruthenium(I) dye in p-type DSCs reported previously (He et al., J. Phys. Chem. C, 2014, 118, 16518) and confirms the effectiveness of a phosphonic acid anchor in the dye and the attachment of the anchoring unit to the pyridine (rather than phenyl) ring of the cyclometallating ligand.
\end{abstract}

\section{Introduction}

There is growing interest in utilizing cyclometallated ruthenium(II) complexes as dyes in dye-sensitized solar cells (DSCs). ${ }^{1-3}$ Typical families of complexes are based upon $\left[\mathrm{Ru}\left(\mathrm{N}^{\wedge} \mathrm{N}\right)_{2}\left(\mathrm{C}^{\wedge} \mathrm{N}\right)\right]^{+}$, $\left[\mathrm{Ru}\left(\mathrm{N}^{\wedge} \mathrm{N}^{\wedge} \mathrm{N}\right)\left(\mathrm{C}^{\wedge} \mathrm{N}^{\wedge} \mathrm{N}\right)\right]^{+}$and $\left[\mathrm{Ru}\left(\mathrm{N}^{\wedge} \mathrm{N}^{\wedge} \mathrm{N}\right)\left(\mathrm{N}^{\wedge} \mathrm{C}^{\wedge} \mathrm{N}\right)\right]^{+}$where archetype $\mathrm{N}^{\wedge} \mathrm{N}, \mathrm{HC}^{\wedge} \mathrm{N}, \mathrm{N}^{\wedge} \mathrm{N}^{\wedge} \mathrm{N}, \mathrm{HC}^{\wedge} \mathrm{N}^{\wedge} \mathrm{N}$ and $\mathrm{N}^{\wedge} \mathrm{CH}^{\wedge} \mathrm{N}$ ligands are, respectively 2, $2^{\prime}$-bipyridine (bpy), 2-phenylpyridine (Hppy), $2,2^{\prime}: 6^{\prime}, 2^{\prime \prime}$-terpyridine (tpy), 6-phenyl-2,2'-bipyridine and 1,3bis(pyridin-2-yl)benzene. The majority of investigations have addressed dyes suited to electron injection at an n-type semiconductor interface, the driving force behind the use of anionic cyclometallating ligands being the replacement of thiocyanate

\footnotetext{
${ }^{a}$ Department of Chemistry, University of Basel, Spitalstrasse 51, CH-4056 Basel, Switzerland. E-mail: catherine.housecroft@unibas.ch

${ }^{b}$ Department of Physics, University of Basel, Klingelbergstrasse 82, CH-4056 Basel, Switzerland

$\dagger$ Electronic supplementary information (ESI) available: Fig. S1-S3: additional NMR spectra. CCDC 1465171. For ESI and crystallographic data in CIF or other electronic format see DOI: 10.1039/c6tc03874c
}

ligands typically present in established Grätzel-type sensitizers. The panchromatic spectral response of $\left[\mathrm{Ru}\left(\mathrm{N}^{\wedge} \mathrm{N}\right)_{2}\left(\mathrm{C}^{\wedge} \mathrm{N}\right)\right]^{+}$dyes in DSCs was identified by Grätzel in 2009. ${ }^{4}$ An advantage of incorporating cyclometallating domains in these complexes is the potential for HOMO-LUMO energy tuning by ligand functionalization, ${ }^{5}$ and photoconversion efficiencies matching or exceeding that of the standard ruthenium dye N719 have been reported. ${ }^{6}$

The HOMO of a $\left[\mathrm{Ru}\left(\mathrm{N}^{\wedge} \mathrm{N}\right)_{2}\left(\mathrm{C}^{\wedge} \mathrm{N}\right)\right]^{+}$complex is localized on the $\mathrm{Ru} / \mathrm{C}^{\wedge} \mathrm{N}$ unit. ${ }^{7}$ Therefore, in cyclometallated dyes for $n$-type DSCs, the $\mathrm{C}^{\wedge} \mathrm{N}$ domain functions as an ancillary ligand and the $\mathrm{N}^{\wedge} \mathrm{N}$ ligand (which contributes to the LUMO) carries the anchoring unit (e.g. carboxylic or phosphonic acid). The first oxidation process is typically shifted $900 \mathrm{mV}$ less positive when compared with the relevant all-nitrogen donor analogue. By switching this functionalization pattern so that the anchor is positioned on the cyclometallating ligand, $\left[\mathrm{Ru}\left(\mathrm{N}^{\wedge} \mathrm{N}\right)_{2}\left(\mathrm{C}^{\wedge} \mathrm{N}\right)\right]^{+}$ and $\left[\mathrm{Ru}\left(\mathrm{N}^{\wedge} \mathrm{N}^{\wedge} \mathrm{N}\right)\left(\mathrm{N}^{\wedge} \mathrm{C}^{\wedge} \mathrm{N}\right)\right]^{+}$complexes become candidates for sensitizers in p-type DSCs (Fig. 1), or at the p-type interface in tandem cells. ${ }^{8}$ This potential has been proven, ${ }^{9-13}$ efficiencies 


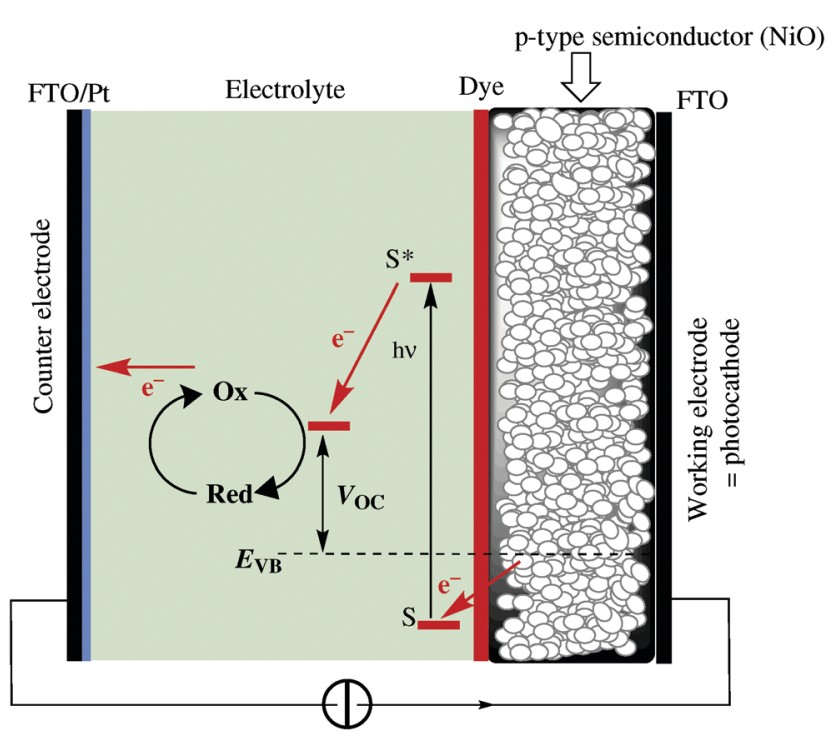

Fig. 1 Working principle of a p-type DSC. S and $\mathrm{S}^{*}$ are the ground and excited states of the dye; $E_{\mathrm{VB}}$ represents the quasi-Fermi level of the valence band of the semiconductor; $V_{\mathrm{OC}}=$ open circuit voltage

are generally low and the area remains ripe for further exploration. It has been demonstrated that tris(bpy)ruthenium(II)- or (bpy)tricarbonylchlororhenium(I)-dyes bearing alkylphosphonate or phosphonic acid anchors bind to $\mathrm{NiO},{ }^{14,15}$ but, to the best of our knowledge, $\left[\mathrm{Ru}\left(\mathrm{N}^{\wedge} \mathrm{N}\right)_{2}\left(\mathrm{C}^{\wedge} \mathrm{N}\right)\right]^{+}$complexes with phosphonic acid anchoring units have not been reported as sensitizers in p-type DSCs. In an initial investigation, ${ }^{16}$ we used the strategy shown in Scheme 1 to access a $\left[\mathrm{Ru}(\mathrm{bpy})_{2}\left(\mathrm{C}^{\wedge} \mathrm{N}\right)\right]^{+}$complex bearing a peripheral phosphonate ester. Although the cyclometallated complex shown in Scheme 1 can be isolated in good yield, its deprotection to yield the corresponding phosphonic acid was, in our hands, unsuccessful. In the present work, we present an alternative synthetic approach, the versatility of which can be used to introduce different anchoring domains into $\left[\mathrm{Ru}\left(\mathrm{N}^{\wedge} \mathrm{N}\right)_{2}\left(\mathrm{C}^{\wedge} \mathrm{N}\right)\right]^{+}$ complexes. We also demonstrate the viability of a phosphonate anchored $\left[\mathrm{Ru}\left(\mathrm{N}^{\wedge} \mathrm{N}\right)_{2}\left(\mathrm{C}^{\wedge} \mathrm{N}\right)\right]^{+}$dye in p-type DSCs.

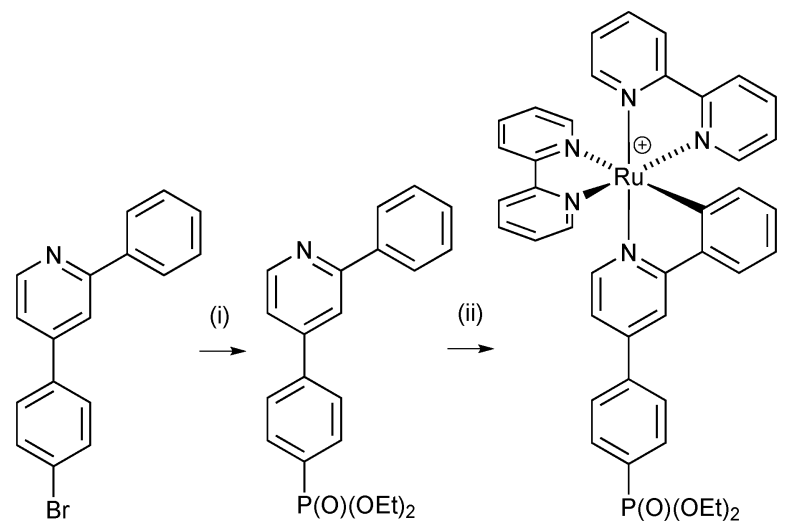

Scheme 1 Previously reported synthesis ${ }^{16}$ of a phosphonate esterfunctionalized $\left[\mathrm{Ru}\left(\mathrm{N}^{\wedge} \mathrm{N}\right)_{2}\left(\mathrm{C}^{\wedge} \mathrm{N}\right)\right]^{+}$complex. Conditions: (i) $\left[\mathrm{Pd}\left(\mathrm{PPh}_{3}\right)_{4}\right], \mathrm{Cs}_{2} \mathrm{CO}_{3}$, $\mathrm{HPO}(\mathrm{OEt})_{2}$, THF, microwave, $110{ }^{\circ} \mathrm{C}, 90 \mathrm{~min}$; (ii) cis- $\left[\mathrm{Ru}(\mathrm{bpy})_{2} \mathrm{Cl}_{2}\right], \mathrm{AgPF}_{6}$.

\section{Experimental}

General

${ }^{1} \mathrm{H},{ }^{13} \mathrm{C},{ }^{11} \mathrm{~B}$ and ${ }^{31} \mathrm{P}$ NMR spectra were recorded on a Bruker Avance III-400 or III-500 spectrometer at $295 \mathrm{~K}$. The ${ }^{1} \mathrm{H}$ and ${ }^{13} \mathrm{C}$ chemical shifts were referenced with respect to residual solvent peaks $\left(\delta \mathrm{TMS}=0\right.$ ), ${ }^{11} \mathrm{~B}$ with respect to $\mathrm{BF}_{3} \cdot \mathrm{Et}_{2} \mathrm{O}$, and ${ }^{31} \mathrm{P}$ with respect to $85 \%$ aqueous $\mathrm{H}_{3} \mathrm{PO}_{4}$. High resolution (HR) ESI-MS were measured on a Bruker maXis $4 \mathrm{G}$ instrument, and LC-ESI-MS using a combination of Shimadzu (LC) and Bruker AmaZon X instruments. An Agilent 8453 spectrophotometer was used to record absorption spectra. Solid-state absorption spectra of dye-functionalized electrodes were recorded using a Cary-5000 spectrophotometer. Microwave reactions were carried out in a Biotage Initiator 8 reactor.

Electrochemical measurements were made with a $\mathrm{CHI} 900 \mathrm{~B}$ instrument using a glassy carbon working electrode, platinum-wire auxiliary electrode, and silver-wire pseudo-reference electrode. Redox potentials were determined by both cyclic and square wave voltammetry. HPLC grade, argon-degassed DMSO or MeCN solutions $\left(\approx 10^{-4} \mathrm{~mol} \mathrm{dm}^{-3}\right)$ of samples were used in the presence of $0.1 \mathrm{M}\left[{ }^{n} \mathrm{Bu}_{4} \mathrm{~N}\right]\left[\mathrm{PF}_{6}\right]$ as supporting electrolyte; the scan rate was $0.1 \mathrm{~V} \mathrm{~s}^{-1}$ and ferrocene $\left(\mathrm{Fc}^{+} / \mathrm{Fc}\right)$ was the internal standard.

\section{Materials}

2-Bromo-4-chloropyridine, 1-bromo-4-iodobenzene, SPhos Pd $\mathrm{G} 2,\left[\mathrm{PdCl}_{2}\left(\mathrm{PPh}_{3}\right)_{2}\right],\left[\mathrm{Pd}\left(\mathrm{PPh}_{3}\right)_{4}\right]$ and $\left[\mathrm{Pd}(\mathrm{dppf}) \mathrm{Cl}_{2}\right]$ were purchased from Sigma-Aldrich; 4-carboxyphenylboronic acid was bought from Acros. All were used as received.

Abbreviation: dppf $=1,1^{\prime}$-bis(diphenylphosphino)ferrocene.

\section{Compound H1}

2-Bromo-4-chloropyridine (1.00 g, $5.20 \mathrm{mmol})$, phenylboronic acid (996 mg, 70\%, $5.7 \mathrm{mmol}),\left[\mathrm{PdCl}_{2}\left(\mathrm{PPh}_{3}\right)_{2}\right]$ (182 mg, $0.300 \mathrm{mmol})$ and $\mathrm{Na}_{2} \mathrm{CO}_{3}(1.10 \mathrm{~g}, 10.4 \mathrm{mmol})$ were suspended in $\mathrm{THF} / \mathrm{H}_{2} \mathrm{O}(1: 1$ by vol, $40 \mathrm{~mL})$. The mixture was heated at $60{ }^{\circ} \mathrm{C}$ for $3 \mathrm{~h}$, and then poured into water $(300 \mathrm{~mL})$ and extracted with $\mathrm{CH}_{2} \mathrm{Cl}_{2}(3 \times 300 \mathrm{~mL})$. The combined organic layers were washed with water $(300 \mathrm{~mL})$, dried over $\mathrm{MgSO}_{4}$ and the solvent was removed under reduced pressure. The resulting yellow oil was purified by column chromatography $\left(\mathrm{SiO}_{2}, \mathrm{EtOAc} /\right.$ cyclohexane 1:10) and $\mathrm{H} 1$ was isolated as a yellowish oil (627 mg, $5.2 \mathrm{mmol}, 64 \%$ ). NMR spectroscopic data were consistent with those reported. ${ }^{17}$

\section{Compound $\mathrm{H} 2$}

Bis(pinacolato)diboron (1.35 g, $5.28 \mathrm{mmol}$ ) and KOAc (777 mg, $7.92 \mathrm{mmol}$ ) were added to a degassed solution of 2-phenyl-4chloropyridine (500 mg, $2.64 \mathrm{mmol}$ ) in dioxane followed by $\left[\mathrm{Pd}(\mathrm{dppf}) \mathrm{Cl}_{2}\right]$ (65 mg, $79 \mu \mathrm{mol}, 3 \mathrm{~mol} \%$ ). The solution was degassed again, and the reaction mixture was heated at reflux for $72 \mathrm{~h}$. After removing the solvent under reduced pressure, the residue was purified by Kugelrohr distillation. The resulting oil was subjected to column chromatography $\left(\mathrm{SiO}_{2}\right.$, EtOAc: cyclohexane 1:2 changing to EtOAc:cyclohexane 5:1) and $\mathrm{H} 2$ was isolated as a brown oil (98 $\mathrm{mg},<348 \mu \mathrm{mol},<13 \%)$. 
The compound could not be completely purified (see text). ${ }^{1} \mathrm{H}$ NMR (500 MHz, $\mathrm{CDCl}_{3}$ ) $\delta / \mathrm{ppm} 8.73(\mathrm{dd}, J=4.7,1.1 \mathrm{~Hz}$, $\left.1 \mathrm{H}, \mathrm{H}^{\mathrm{A} 6}\right), 8.12\left(\mathrm{~s}, 1 \mathrm{H}, \mathrm{H}^{\mathrm{A} 3}\right), 8.05\left(\mathrm{~m}, 2 \mathrm{H}, \mathrm{H}^{\mathrm{B} 2}\right), 7.59(\mathrm{dd}, J=4.8$, $1.1 \mathrm{~Hz}, 1 \mathrm{H}, \mathrm{H}^{\mathrm{A} 5}$ ), 7.48 (ddt, $J=8.6,7.2,1.8 \mathrm{~Hz}, 2 \mathrm{H}, \mathrm{H}^{\mathrm{B} 3}$ ), 7.43 $\left(\mathrm{m}, 1 \mathrm{H}, \mathrm{H}^{\mathrm{B} 4}\right) .{ }^{13} \mathrm{C}\left\{{ }^{1} \mathrm{H}\right\}$ NMR $\left(126 \mathrm{MHz}, \mathrm{CDCl}_{3}\right) \delta / \mathrm{ppm} 156.7$ $\left(\mathrm{C}^{\mathrm{A} 2}\right), 149.0\left(\mathrm{C}^{\mathrm{A} 6}\right), 139.4\left(\mathrm{C}^{\mathrm{B} 1}\right), 128.8\left(\mathrm{C}^{\mathrm{B} 4}\right), 128.6\left(\mathrm{C}^{\mathrm{B} 3}\right), 127.1$ $\left(\mathrm{C}^{\mathrm{A} 5}\right), 127.0\left(\mathrm{C}^{\mathrm{B} 2}\right), 125.7\left(\mathrm{C}^{\mathrm{A} 3}\right), 84.5\left(\mathrm{C}^{\mathrm{Me}}\right) .{ }^{11} \mathrm{~B} \mathrm{NMR}(128 \mathrm{MHz}$, $\left.\mathrm{CDCl}_{3}\right) \delta / \mathrm{ppm} 30.8$.

\section{$\left[\mathrm{Ru}(\mathrm{bpy})_{2}(\mathbf{1})\right]\left[\mathrm{PF}_{6}\right]$}

A solution of $\left[\mathrm{Ru}(\mathrm{bpy})_{2} \mathrm{Cl}_{2}\right](581 \mathrm{mg}, 1.20 \mathrm{mmol}), \mathrm{H1}$ (501 mg, $2.64 \mathrm{mmol}$ ) and $\mathrm{AgPF}_{6}(667 \mathrm{mg}, 2.64 \mathrm{mmol})$ in $\mathrm{CH}_{2} \mathrm{Cl}_{2}(75 \mathrm{~mL})$ was heated at reflux for $19 \mathrm{~h}$. The reaction mixture was filtered over celite and washed thoroughly with $\mathrm{CH}_{2} \mathrm{Cl}_{2}$. The solvent was removed under vacuum and the dark purple residue was purified by column chromatography (alumina, acetone/ pentane $2: 1$, changing to acetone). After solvent evaporation, an oil was isolated. The oil was diluted with $\mathrm{CH}_{2} \mathrm{Cl}_{2}$ and then hexanes were added to precipitate the product. After filtration, $\left[\mathrm{Ru}(\mathrm{bpy})_{2}(\mathbf{1})\right]\left[\mathrm{PF}_{6}\right]$ was isolated as a dark purple solid (584 mg, $0.782 \mathrm{mmol}, 65.2 \%) .{ }^{1} \mathrm{H}$ NMR (500 $\left.\mathrm{MHz}, \mathrm{CD}_{3} \mathrm{CN}\right) \delta / \mathrm{ppm} 8.47$ $\left(\mathrm{dt}, J=8.3,1.0 \mathrm{~Hz}, 1 \mathrm{H}, \mathrm{H}^{\mathrm{A} 3}\right), 8.40\left(\mathrm{dt}, J=8.3,1.0 \mathrm{~Hz}, 1 \mathrm{H}, \mathrm{H}^{\mathrm{B} 3}\right)$, 8.32 (overlapping $\mathrm{m}, 1 \mathrm{H}, \mathrm{H}^{\mathrm{C} 3+\mathrm{D} 3}$ ), $8.07\left(\mathrm{~d}, J=2.3 \mathrm{~Hz}, 1 \mathrm{H}, \mathrm{H}^{\mathrm{E} 3}\right.$ ), 8.03 (ddd, $J=5.7,1.6,0.8 \mathrm{~Hz}, 1 \mathrm{H}, \mathrm{H}^{\mathrm{B} 6}$ ), 8.00 (ddd, $J=8.2,7.6$, $1.6 \mathrm{~Hz}, 1 \mathrm{H}, \mathrm{H}^{\mathrm{A} 4}$ ), 7.88-7.82 (overlapping $\mathrm{m}, 5 \mathrm{H}, \mathrm{H}^{\mathrm{F} 3+\mathrm{A} 6+\mathrm{B} 4+\mathrm{C} 4+\mathrm{D} 6}$ ), 7.81 (ddd, $\left.J=8.2,7.5,1.4 \mathrm{~Hz}, 1 \mathrm{H}, \mathrm{H}^{\mathrm{D} 4}\right), 7.72(\mathrm{ddd}, J=5.7,1.5$, $0.8 \mathrm{~Hz}, 1 \mathrm{H}, \mathrm{H}^{\mathrm{C} 6}$ ), 7.51 (dd, $J=6.2,0.6 \mathrm{~Hz}, 1 \mathrm{H}, \mathrm{H}^{\mathrm{E} 6}$ ), 7.43 (ddd, $J=7.6,5.4,1.2 \mathrm{~Hz}, 1 \mathrm{H}, \mathrm{H}^{\mathrm{A} 5}$ ), 7.26-7.20 (overlapping $\mathrm{m}, 3 \mathrm{H}$, $\left.\mathrm{H}^{\mathrm{B} 5+\mathrm{C} 5+\mathrm{D} 5}\right), 6.97\left(\mathrm{dd}, J=6.2,2.3 \mathrm{~Hz}, 1 \mathrm{H}, \mathrm{H}^{\mathrm{E} 5}\right), 6.91(\mathrm{td}, J=7.4$, $\left.1.4 \mathrm{~Hz}, 1 \mathrm{H}, \mathrm{H}^{\mathrm{F} 4}\right), 6.86\left(\mathrm{td}, J=7.2,1.4 \mathrm{~Hz}, 1 \mathrm{H}, \mathrm{H}^{\mathrm{F} 5}\right), 6.46(\mathrm{~m}, 1 \mathrm{H}$, $\left.\mathrm{H}^{\mathrm{F} 6}\right) .{ }^{13} \mathrm{C}\left\{{ }^{1} \mathrm{H}\right\}$ NMR (126 MHz, CD $\left.{ }_{3} \mathrm{CN}\right) \delta / \mathrm{ppm} 194.7\left(\mathrm{C}^{\mathrm{F} 1}\right)$, $169.6\left(\mathrm{C}^{\mathrm{E} 2}\right), 158.5\left(\mathrm{C}^{\mathrm{B} 2}\right), 157.7\left(\mathrm{C}^{\mathrm{C} 2}\right), 157.6\left(\mathrm{C}^{\mathrm{D} 2}\right), 156.0\left(\mathrm{C}^{\mathrm{A} 2}\right)$, $155.0\left(\mathrm{C}^{\mathrm{B} 6}\right), 152.2\left(\mathrm{C}^{\mathrm{E} 6}\right), 151.3\left(\mathrm{C}^{\mathrm{A} 6 / \mathrm{D} 6}\right), 150.9\left(\mathrm{C}^{\mathrm{C} 6}\right), 150.1$ $\left(\mathrm{C}^{\mathrm{A} 6 / \mathrm{D} 6}\right), 145.8\left(\mathrm{C}^{\mathrm{F} 2}\right), 143.9\left(\mathrm{C}^{\mathrm{E} 4}\right), 137.4\left(\mathrm{C}^{\mathrm{A} 4}\right), 136.5\left(\mathrm{C}^{\mathrm{F} 6}\right), 136.0$ $\left(\mathrm{C}^{\mathrm{B} 4 / \mathrm{C} 4}\right), 134.9\left(\mathrm{C}^{\mathrm{B} 4 / \mathrm{C} 4}\right), 134.7\left(\mathrm{C}^{\mathrm{D} 4}\right), 129.7\left(\mathrm{C}^{\mathrm{F} 5}\right), 128.0\left(\mathrm{C}^{\mathrm{A} 5}\right)$, $127.3\left(\mathrm{C}^{\mathrm{B} 5 / \mathrm{C} 5 / \mathrm{D} 5}\right), 127.2\left(\mathrm{C}^{\mathrm{B} 5 / \mathrm{C} 5 / \mathrm{D} 5}\right), 127.0\left(\mathrm{C}^{\mathrm{B} 5 / \mathrm{C} 5 / \mathrm{D} 5}\right), 125.5\left(\mathrm{C}^{\mathrm{F} 3}\right)$, 124.4 $\left(\mathrm{C}^{\mathrm{B} 3}\right), 124.1\left(\mathrm{C}^{\mathrm{A} 3}\right), 123.9\left(\mathrm{C}^{\mathrm{C} 3+\mathrm{D} 3}\right), 123.1\left(\mathrm{C}^{\mathrm{E} 5}\right), 122.0\left(\mathrm{C}^{\mathrm{F} 4}\right)$, $119.8\left(\mathrm{C}^{\mathrm{E} 3}\right) .{ }^{31} \mathrm{P}\left\{{ }^{1} \mathrm{H}\right\}$ NMR $\left(162 \mathrm{MHz}, \mathrm{CDCl}_{3}\right) \delta / \mathrm{ppm}-144.6$ (septet, $J=707 \mathrm{~Hz}, \mathrm{PF}_{6}$ ). LC-ESI-MS $m / z 602.1\left[\mathrm{M}-\mathrm{PF}_{6}\right]^{+}$(calc. 602.1). UV-Vis (MeOH, $\left.1 \times 10^{-5} \mathrm{M}\right) \lambda / \mathrm{nm}\left(\varepsilon / \mathrm{dm}^{3} \mathrm{~mol}^{-1} \mathrm{~cm}^{-1}\right)$ 249 (36 000), 296 (62 000), 369 (12 000), 409 (11 000), 491 (9800), 540 (10 000). Found C 49.12, H 3.47, N 9.52; $\mathrm{C}_{31} \mathrm{H}_{23} \mathrm{ClF}_{6} \mathrm{~N}_{5} \mathrm{PRu}$. $0.5 \mathrm{H}_{2} \mathrm{O}$ requires $\mathrm{C} 49.25, \mathrm{H} 3.20, \mathrm{~N} 9.26$.

\section{Diethyl 4-bromobenzenephosphonate}

1-Bromo-4-iodobenzene $(2.00 \mathrm{~g}, 7.10 \mathrm{mmol}), \quad\left[\mathrm{Pd}\left(\mathrm{PPh}_{3}\right)_{4}\right]$ (408 mg, $0.354 \mathrm{mmol}$ ) and $\mathrm{Cs}_{2} \mathrm{CO}_{3}(4.84 \mathrm{~g}, 14.80 \mathrm{mmol})$ were combined in anhydrous THF $(10 \mathrm{~mL})$. The mixture was degassed for $10 \mathrm{~min}$ and heated at reflux for $2.5 \mathrm{~h}$. The reaction mixture was filtered over celite and washed thoroughly with THF. After removal of solvent, the residue was purified by flash column chromatography $\left(\mathrm{SiO}_{2}, \mathrm{CH}_{2} \mathrm{Cl}_{2}\right.$, changing to $\mathrm{CH}_{2} \mathrm{Cl}_{2} / \mathrm{EtOH}$ $100: 3)$. Diethyl 4-bromobenzenephosphonate was isolated as a yellow oil (1.5 g, $7.1 \mathrm{mmol}, 73 \%)$. NMR spectroscopic data were consistent with the literature. ${ }^{18}$

\section{Compound 3}

$\left[\mathrm{Pd}(\mathrm{dppf}) \mathrm{Cl}_{2}\right](70 \mathrm{mg}, 85 \mu \mathrm{mol})$ was added to a degassed mixture of diethyl 4-bromobenzenephosphonate (500 mg, $1.7 \mathrm{mmol}$ ), bis(pinacolato)diboron (877 mg, $3.4 \mathrm{mmol}$ ) and KOAc $(503 \mathrm{mg}$, $5.1 \mathrm{mmol})$ in THF $(20 \mathrm{~mL})$. The reaction mixture was degassed again and heated in a microwave reactor at $90{ }^{\circ} \mathrm{C}$ for $1 \mathrm{~h}$. The mixture was then filtered over celite and the solvent was removed from the filtrate. The resulting brown oil was purified by column chromatography $\left(\mathrm{SiO}_{2}, \mathrm{CH}_{2} \mathrm{Cl}_{2}\right.$, changing to $\mathrm{CH}_{2} \mathrm{Cl}_{2}$ / $\mathrm{EtOH}, 100: 5)$ and used in the next step without further purification. The ${ }^{1} \mathrm{H}$ NMR spectroscopic data matched those reported. ${ }^{19}{ }^{31} \mathrm{P}\left\{{ }^{1} \mathrm{H}\right\}$ NMR (162 $\left.\mathrm{MHz}, \mathrm{CDCl}_{3}\right) \delta / \mathrm{ppm}+18.6$. ${ }^{11} \mathrm{~B}$ NMR $\left(128 \mathrm{MHz}, \mathrm{CDCl}_{3}\right) \delta / \mathrm{ppm}+22.4$.

\section{Compound $\mathrm{H}_{2} 4$}

A solution of $3(582 \mathrm{mg}, 1.7 \mathrm{mmol})$, and $\mathrm{Me}_{3} \mathrm{SiBr}(2.8 \mathrm{~mL}$, $21.4 \mathrm{mmol}$ ) in dry $\mathrm{CH}_{2} \mathrm{Cl}_{2}$ was stirred at room temperature for $19 \mathrm{~h}$. The reaction was quenched with water and the layers separated. The aqueous layer was evaporated to dryness and the residue was redissolved in EtOH. The solvent was evaporated under reduced pressure. The product was recrystallized from $\mathrm{MeO}^{t} \mathrm{Bu}$ and $\mathrm{H}_{2} 4$ was isolated as a white solid $(276 \mathrm{mg}$, $1.1 \mathrm{mmol}, 65 \%) .{ }^{1} \mathrm{H}$ NMR (400 MHz, DMSO- $\left.d_{6}\right) \delta / \mathrm{ppm} 10.90$ $\left(\mathrm{s}, 2 \mathrm{H}, \mathrm{H}^{\mathrm{POH}}\right), 7.78-7.63\left(\mathrm{~m}, 4 \mathrm{H}, \mathrm{H}^{2+3}\right), 1.30\left(\mathrm{~s}, 12 \mathrm{H}, \mathrm{H}^{\mathrm{Me}}\right)$. ${ }^{13} \mathrm{C}\left\{{ }^{1} \mathrm{H}\right\}$ NMR (126 MHz, DMSO- $\left.d_{6}\right) \delta /$ ppm $137.2\left(\mathrm{~d}, J_{\mathrm{PC}}=\right.$ $\left.179 \mathrm{~Hz}, \mathrm{C}^{1}\right), 134.0$ (d, $\left.J=13.8 \mathrm{~Hz}, \mathrm{C}^{3}\right), 129.8$ (d, $J=9.4 \mathrm{~Hz}, \mathrm{C}^{2}$ ), $83.9\left(\mathrm{C}^{\mathrm{q}-\mathrm{pin}}\right), 24.7\left(\mathrm{C}^{\mathrm{Me}}\right) .{ }^{31} \mathrm{P}\left\{{ }^{1} \mathrm{H}\right\}$ NMR (162 MHz, DMSO- $\left.d_{6}\right)$ $\delta / \mathrm{ppm}+12.2$. ${ }^{11} \mathrm{~B}$ NMR (128 MHz, DMSO- $\left.d_{6}\right) \delta / \mathrm{ppm}+28.4$. LC-ESI-MS $m / z: 285.1[\mathrm{M}+\mathrm{H}]^{+}$(calc. 285.1). Found C 49.87, $\mathrm{H}$ 6.43; $\mathrm{C}_{12} \mathrm{H}_{18} \mathrm{BO}_{5} \mathrm{P} \cdot 0.25 \mathrm{H}_{2} \mathrm{O}$ requires: $\mathrm{C} 49.95, \mathrm{H} 6.46$.

\section{$\left[\mathrm{Ru}(\mathrm{bpy})_{2}(\mathrm{H} 5)\right]$}

$\left[\mathrm{Ru}(\mathrm{bpy})_{2}(\mathbf{1})\right]\left[\mathrm{PF}_{6}\right](100 \mathrm{mg}, 134 \mu \mathrm{mol}), \mathrm{H}_{2} 4$ (67 mg, $\left.268 \mu \mathrm{mol}\right)$, $\mathrm{K}_{3} \mathrm{PO}_{4}(199 \mathrm{mg}, 938 \mu \mathrm{mol})$ and SPhos Pd G2 $(4.8 \mathrm{mg}, 6.7 \mu \mathrm{mol}$, $5 \mathrm{~mol} \%)$ were dissolved in degassed $\mathrm{MeCN} / \mathrm{H}_{2} \mathrm{O}(1: 1,40 \mathrm{~mL})$. The mixture was degassed again and heated at $70{ }^{\circ} \mathrm{C}$ for $5 \mathrm{~h}$. The solvent was then removed under reduced pressure and additional water was added to yield a precipitate. This was collected over celite, washed with water and dissolved in $\mathrm{MeOH}$. After removing the solvent, the resulting red solid was purified by column chromatography $\left(\mathrm{SiO}_{2}, \mathrm{MeCN} /\right.$ saturated aqueous $\mathrm{KNO}_{3} /$ $\mathrm{H}_{2} \mathrm{O} 7: 1: 0.5$, changing to MeCN/saturated aqueous $\mathrm{KNO}_{3} / \mathrm{H}_{2} \mathrm{O}$ $7: 2: 2$ ). The MeCN was evaporated and additional water was added. The precipitate that formed was collected over celite, washed thoroughly with water and dissolved with $\mathrm{MeOH}$. After evaporating the solvent under reduced pressure, $\left[\mathrm{Ru}(\mathrm{bpy})_{2}(\mathrm{H} 5)\right]$ was isolated as a dark red solid ( $82 \mathrm{mg}, 113 \mu \mathrm{mol}, 84 \%) .{ }^{1} \mathrm{H} \mathrm{NMR}$ $\left(500 \mathrm{MHz}, \mathrm{CD}_{3} \mathrm{OD}\right) \delta / \mathrm{ppm} 8.62\left(\mathrm{dt}, J=8.3,1.0 \mathrm{~Hz}, 1 \mathrm{H}, \mathrm{H}^{\mathrm{A} 3}\right), 8.53$ $\left(\mathrm{dt}, J=8.2,1.1 \mathrm{~Hz}, 1 \mathrm{H}, \mathrm{H}^{\mathrm{B} 3}\right), 8.46\left(\mathrm{dt}, J=8.3,1.1 \mathrm{~Hz}, 1 \mathrm{H}, \mathrm{H}^{\mathrm{C} 3}\right)$, $8.44\left(\mathrm{dt}, J=8.2,1.1 \mathrm{~Hz}, 1 \mathrm{H}, \mathrm{H}^{\mathrm{D} 3}\right), 8.32\left(\mathrm{~d}, J=1.9 \mathrm{~Hz}, 1 \mathrm{H}, \mathrm{H}^{\mathrm{E} 3}\right)$, 8.15 (ddd, $J=5.7,1.5,0.7 \mathrm{~Hz}, 1 \mathrm{H}, \mathrm{H}^{\mathrm{B} 6}$ ), 8.07-7.98 (overlapping $\mathrm{m}$, $2 \mathrm{H}, \mathrm{H}^{\mathrm{A} 4+\mathrm{F} 3}$ ), 7.98-7.88 (overlapping $\mathrm{m}, 4 \mathrm{H}, \mathrm{H}^{\mathrm{G} 2+\mathrm{D} 6+\mathrm{A} 6}$ ), 7.89-7.73 (overlapping $\mathrm{m}, 6 \mathrm{H}, \mathrm{H}^{\mathrm{C} 4+\mathrm{B} 4+\mathrm{D} 4+\mathrm{G} 3+\mathrm{C} 6}$ ), 7.61 (dd, $J=6.0,0.6 \mathrm{~Hz}$, $1 \mathrm{H}, \mathrm{H}^{\mathrm{E} 6}$ ), 7.48 (ddd, $J=7.6,5.4,1.2 \mathrm{~Hz}, 1 \mathrm{H}, \mathrm{H}^{\mathrm{A} 5}$ ), 7.30-7.20 (overlapping $\mathrm{m}, 4 \mathrm{H}, \mathrm{H}^{\mathrm{B} 5+\mathrm{C} 5+\mathrm{D} 5+\mathrm{E} 5}$ ), 6.92 (ddd, $J=7.7,7.1,1.3 \mathrm{~Hz}$, 
$\left.1 \mathrm{H}, \mathrm{H}^{\mathrm{F} 4}\right), 6.82\left(\mathrm{td}, J=7.3,1.3 \mathrm{~Hz}, 1 \mathrm{H}, \mathrm{H}^{\mathrm{F} 5}\right), 6.44(\mathrm{dd}, J=7.4$, $\left.0.9 \mathrm{~Hz}, 2 \mathrm{H}, \mathrm{H}^{\mathrm{F} 6}\right) .{ }^{13} \mathrm{C}\left\{{ }^{1} \mathrm{H}\right\}$ NMR (126 MHz, CD $\left.\mathrm{OD}\right) \delta / \mathrm{ppm} 193.7$ $\left(\mathrm{C}^{\mathrm{F} 1}\right), 169.4\left(\mathrm{C}^{\mathrm{E} 2}\right), 159.2\left(\mathrm{C}^{\mathrm{B} 2}\right), 158.5\left(\mathrm{C}^{\mathrm{C} 2}\right), 158.2\left(\mathrm{C}^{\mathrm{D} 2}\right), 156.8$ $\left(\mathrm{C}^{\mathrm{A} 2}\right), 155.5\left(\mathrm{C}^{\mathrm{B} 6}\right), 151.4\left(\mathrm{C}^{\mathrm{D} 6}\right), 151.3\left(\mathrm{C}^{\mathrm{E} 6}\right), 150.9\left(\mathrm{C}^{\mathrm{C} 6}\right), 150.1$ $\left(\mathrm{C}^{\mathrm{A} 6}\right), 148.9\left(\mathrm{C}^{\mathrm{E} 4}\right), 146.8\left(\mathrm{C}^{\mathrm{F} 2}\right), 139.5\left(\mathrm{C}^{\mathrm{G} 4}\right), 137.5\left(\mathrm{C}^{\mathrm{A} 4}\right), 136.4\left(\mathrm{C}^{\mathrm{F} 6}\right)$, $136.2\left(\mathrm{C}^{\mathrm{B} 4}\right), 134.9\left(\mathrm{C}^{\mathrm{C} 4}\right), 132.8\left(\mathrm{C}^{\mathrm{D} 4}\right), 129.7\left(\mathrm{C}^{\mathrm{G} 2}\right), 128.2\left(\mathrm{C}^{\mathrm{F} 5}\right)$, $127.4\left(\mathrm{C}^{\mathrm{A} 5}\right), 127.3\left(\mathrm{C}^{\mathrm{B} 5+\mathrm{C} 5+\mathrm{D} 5}\right), 127.2\left(\mathrm{C}^{\mathrm{G} 3}\right), 125.6\left({ }^{\mathrm{CF} 3}\right), 124.6\left(\mathrm{C}^{\mathrm{B} 3}\right)$, $124.5\left(\mathrm{C}^{\mathrm{A} 3}\right), 124.1\left(\mathrm{C}^{\mathrm{C} 3+\mathrm{D} 3}\right), 122.1\left(\mathrm{C}^{\mathrm{F} 4}\right), 121.1\left(\mathrm{C}^{\mathrm{E} 5}\right), 117.3\left(\mathrm{C}^{\mathrm{E} 3}\right)$. ${ }^{31} \mathrm{P}\left\{{ }^{1} \mathrm{H}\right\}$ NMR $\left(162 \mathrm{MHz}, \mathrm{CD}_{3} \mathrm{OD}\right) \delta / \mathrm{ppm}+10.8$. LC-ESI-MS $\mathrm{m} / \mathrm{z}$ : $724.2[\mathrm{M}+\mathrm{H}]^{+}$(calc. 724.1). HR ESI-MS m/z: 724.1068 (calc. 724.1056). UV-Vis (MeOH, $\left.1 \times 10^{-5} \mathrm{M}\right) \lambda / \mathrm{nm}\left(\varepsilon / \mathrm{dm}^{3} \mathrm{~mol}^{-1} \mathrm{~cm}^{-1}\right)$ : 251 (47 000), 287 (sh, 59000$), 296$ (66 000), 374 (13 000), 420 (16000), 492 (12000), 544 (12000). Found C 57.96, H 4.47, $\mathrm{N} 9.41 ; \mathrm{C}_{37} \mathrm{H}_{28} \mathrm{~N}_{5} \mathrm{O}_{3} \mathrm{PRu} \cdot 2.5 \mathrm{H}_{2} \mathrm{O}$ requires C 57.88, $\mathrm{H}$ 4.33, N 9.12.

\section{$\left[\mathrm{Ru}(\mathrm{bpy})_{2}(\mathrm{H} 6)\right]\left[\mathrm{PF}_{6}\right]$}

$\left[\mathrm{Ru}(\mathrm{bpy})_{2}(\mathbf{1})\right]\left[\mathrm{PF}_{6}\right](50 \mathrm{mg}, 67 \mu \mathrm{mol}), 4$-carboxyphenylboronic acid $(22.2 \mathrm{mg}, 134 \mu \mathrm{mol}), \mathrm{K}_{3} \mathrm{PO}_{4}(99.4 \mathrm{mg}, 468 \mu \mathrm{mol})$ and SPhos Pd G2 $(2.4 \mathrm{mg}, 3.3 \mu \mathrm{mol}, 5 \mathrm{~mol} \%)$ were dissolved in degassed $\mathrm{MeCN} / \mathrm{H}_{2} \mathrm{O}(1: 1,40 \mathrm{~mL})$. The mixture was degassed again and heated at $70{ }^{\circ} \mathrm{C}$ for $5 \mathrm{~h}$. Then, the $\mathrm{MeCN}$ was evaporated and additional water was added to precipitate the crude product. The precipitate was collected over celite, washed with water and dissolved with $\mathrm{MeOH}$. Solvent was removed and the red residue was purified by column chromatography $\left(\mathrm{SiO}_{2}, \mathrm{MeCN} /\right.$ saturated aqueous $\left.\mathrm{KNO}_{3} / \mathrm{H}_{2} \mathrm{O}, 7: 1: 0.5\right)$. The $\mathrm{MeCN}$ was evaporated, additional water was added and the precipitate that formed was filtered over celite, and washed with water. The solid was dissolved in aqueous $\mathrm{MeOH}$; enough water was used to render the compound soluble. An excess of aqueous $\mathrm{NH}_{4} \mathrm{PF}_{6}$ was added and the red precipitate was collected by filtration. $\left[\mathrm{Ru}(\mathrm{bpy})_{2}(\mathrm{H6})\right]\left[\mathrm{PF}_{6}\right]$ was isolated as a dark red solid $(47 \mathrm{mg}$, $56 \mu \mathrm{mol}, 84 \%) .{ }^{1} \mathrm{H}$ NMR (500 MHz, acetone- $\left.d_{6}\right) \delta / \mathrm{ppm} 8.76$ $\left(\mathrm{d}, J=8.4 \mathrm{~Hz}, 1 \mathrm{H}, \mathrm{H}^{\mathrm{A} 3}\right), 8.68\left(\mathrm{~d}, J=8.3 \mathrm{~Hz}, 1 \mathrm{H}, \mathrm{H}^{\mathrm{B} 3}\right), 8.61$ $\left(\mathrm{d}, J=8.3 \mathrm{~Hz}, 1 \mathrm{H}, \mathrm{H}^{\mathrm{C} 3}\right), 8.58\left(\mathrm{~d}, J=8.1 \mathrm{~Hz}, 1 \mathrm{H}, \mathrm{H}^{\mathrm{D} 3}\right), 8.52(\mathrm{~d}$, $\left.J=2.0 \mathrm{~Hz}, 1 \mathrm{H}, \mathrm{H}^{\mathrm{E} 3}\right), 8.19\left(\mathrm{~m}, 1 \mathrm{H}, \mathrm{H}^{\mathrm{B} 6 / \mathrm{C} 6 / \mathrm{D} 6}\right), 8.18-8.12$ (overlapping $\left.\mathrm{m}, 4 \mathrm{H}, \mathrm{H}^{\mathrm{G} 2+\mathrm{A} 4+\mathrm{F} 3}\right), 8.07\left(\mathrm{~m}, 1 \mathrm{H}, \mathrm{H}^{\mathrm{A} 6}\right), 8.04\left(\mathrm{~m}, 1 \mathrm{H}, \mathrm{H}^{\mathrm{B} 6 / \mathrm{C} 6 / \mathrm{D} 6}\right), 7.99-$ 7.93 (overlapping $\mathrm{m}, 4 \mathrm{H}, \mathrm{H}^{\mathrm{B} 6 / \mathrm{C} 6 / \mathrm{D} 6+\mathrm{G} 3+\mathrm{B} 4}$ ), 7.93-7.88 (overlapping $\left.\mathrm{m}, 2 \mathrm{H}, \mathrm{H}^{\mathrm{C} 4+\mathrm{D} 4}\right), 7.79\left(\mathrm{~d}, J=5.7 \mathrm{~Hz}, 1 \mathrm{H}, \mathrm{H}^{\mathrm{E} 6}\right), 7.60$ (ddd, $J=7.5,5.6,1.2 \mathrm{~Hz}, 1 \mathrm{H}, \mathrm{H}^{\mathrm{A} 5}$ ), 7.42-7.35 (overlapping $\mathrm{m}, 4 \mathrm{H}$, $\left.\mathrm{H}^{\mathrm{E} 5+\mathrm{B} 5+\mathrm{C} 5+\mathrm{D} 5}\right), 6.92\left(\mathrm{td}, J=7.4,1.3 \mathrm{~Hz}, 1 \mathrm{H}, \mathrm{H}^{\mathrm{F} 4}\right), 6.85(\mathrm{td}, J=7.3$, $\left.1.3 \mathrm{~Hz}, 1 \mathrm{H}, \mathrm{H}^{\mathrm{F} 5}\right), 6.52\left(\mathrm{dd}, J=7.8,1.3 \mathrm{~Hz}, 1 \mathrm{H}, \mathrm{H}^{\mathrm{F} 6}\right) .{ }^{13} \mathrm{C}\left\{{ }^{1} \mathrm{H}\right\} \mathrm{NMR}$ $\left(126 \mathrm{MHz}\right.$, acetone- $\left.d_{6}\right) \delta / \mathrm{ppm} 170.0\left(\mathrm{C}^{\mathrm{E} 2}\right), 158.0\left(\mathrm{C}^{\mathrm{C} 2+\mathrm{D} 2}\right), 155.5$ $\left(\mathrm{C}^{\mathrm{B} 6 / \mathrm{C} 6 / \mathrm{D} 6}\right), 151.5\left(\mathrm{C}^{\mathrm{A6}}\right), 151.3\left(\mathrm{C}^{\mathrm{B} 6 / \mathrm{C} 6 / \mathrm{D} 6}\right), 150.8\left(\mathrm{C}^{\mathrm{B} 6 / \mathrm{C} 6 / \mathrm{D} 6}\right), 150.1$ $\left(\mathrm{C}^{\mathrm{E} 6}\right), 137.4\left(\mathrm{C}^{\mathrm{A} 4}\right), 136.1\left(\mathrm{C}^{\mathrm{F} 6}\right), 135.5\left(\mathrm{C}^{\mathrm{B} 4 / \mathrm{C} 4 / \mathrm{D} 4}\right), 134.7\left(\mathrm{C}^{\mathrm{B} 4 / \mathrm{C} 4 / \mathrm{D} 4}\right)$, $134.4\left(\mathrm{C}^{\mathrm{B} 4 / \mathrm{C} 4 / \mathrm{D} 4}\right), 131.1\left(\mathrm{C}^{\mathrm{G} 2}\right), 129.2\left(\mathrm{C}^{\mathrm{F} 5}\right), 128.0\left(\mathrm{C}^{\mathrm{A} 5}\right), 127.7$ $\left(\mathrm{C}^{\mathrm{G} 3}\right), 127.2\left(\mathrm{C}^{\mathrm{B} 5+\mathrm{C} 5+\mathrm{D} 5}\right), 125.4\left(\mathrm{C}^{\mathrm{F} 3}\right), 124.3\left(\mathrm{C}^{\mathrm{B} 3}\right), 124.1\left(\mathrm{C}^{\mathrm{A} 3}\right)$, $123.9\left(\mathrm{C}^{\mathrm{C} 3+\mathrm{D} 3}\right), 121.3\left(\mathrm{C}^{\mathrm{F} 4}\right), 120.7\left(\mathrm{C}^{\mathrm{E} 5}\right), 117.0\left(\mathrm{C}^{\mathrm{E} 3}\right)$; signals for $\mathrm{C}^{\mathrm{COOH}}, \mathrm{C}^{\mathrm{B} 2}, \mathrm{C}^{\mathrm{A} 2}, \mathrm{C}^{\mathrm{E} 4}, \mathrm{C}^{\mathrm{F} 2}, \mathrm{C}^{\mathrm{G} 4}$ not resolved. ${ }^{31} \mathrm{P}\left\{{ }^{1} \mathrm{H}\right\}$ NMR $\left(202 \mathrm{MHz}\right.$, acetone- $\left.d_{6}\right) \delta / \mathrm{ppm}-144.7\left(J_{\mathrm{PF}}=727 \mathrm{~Hz}\right)$. LC-ESIMS positive mode: $m / z: 688.1\left[\mathrm{M}-\mathrm{PF}_{6}\right]^{+}$(calc. 688.1). ESI-MS negative mode: $145.0\left[\mathrm{PF}_{6}\right]^{-}$(calc. 145.0). HR ESI-MS positive mode: $m / z: 688.1283\left[\mathrm{M}-\mathrm{PF}_{6}\right]^{+}$(calc. 688.1286). UV-Vis (MeOH, $\left.1 \times 10^{-5} \mathrm{M}\right) \lambda / \mathrm{nm}\left(\varepsilon / \mathrm{dm}^{3} \mathrm{~mol}^{-1} \mathrm{~cm}^{-1}\right) 252$ (47 000), 296 (69 000), 374 (13 000), 427 (16 000), 492 (12 000), 543 (12 000). Satisfactory elemental analysis could not be obtained.

\section{Crystallography}

Single crystal data were collected on a Bruker APEX-II diffractometer; data reduction, solution and refinement used APEX2, SuperFlip and CRYSTALS respectively. ${ }^{20-22}$ Structure analysis used Mercury v. 3.6. ${ }^{23,24}$

\section{$\left[\mathbf{R u}(\mathbf{b p y})_{2}(\mathbf{1})\right]\left[\mathrm{PF}_{6}\right]$}

$\mathrm{C}_{31} \mathrm{H}_{23} \mathrm{ClF}_{6} \mathrm{~N}_{5} \mathrm{PRu}, M=747.04$, purple needle, monoclinic, space group $P 2_{1} / n, a=12.9459(8), b=17.2178(10), c=14.0795(8) \AA$, $\beta=110.666(2)^{\circ}, U=2936.38(17) \AA^{3}, Z=4, D_{\mathrm{c}}=1.690 \mathrm{Mg} \mathrm{m}^{-3}$, $\mu(\mathrm{Cu}-\mathrm{K} \alpha)=6.304 \mathrm{~mm}^{-1}, T=123 \mathrm{~K}$. Total 27090 reflections, 5389 unique, $R_{\text {int }}=0.038$. Refinement of 4765 reflections (406 parameters) with $I>2 \sigma(I)$ converged at final $R_{1}=0.0257$ $\left(R_{1}\right.$ all data $\left.=0.0311\right), \mathrm{w} R_{2}=0.0266\left(\mathrm{w} R_{2}\right.$ all data $\left.=0.0360\right), \mathrm{gof}=$ 1.1321. CCDC 1465171 .

\section{Electrode preparation and device assembly}

Each working electrode was prepared from an FTO glass plate (SolaronixTCO22-7, $2.2 \mathrm{~mm}$ thickness, sheet resistance $\approx 7 \Omega$ square $^{-1}$ ) which was cleaned by sonicating in Sonoswiss surfactant ( $2 \%$ in milliQ water), and rinsed with milliQ water and EtOH. The surface was activated in a UV-O ${ }_{3}$ system (Model 256-220, Jelight Company Inc.) for $20 \mathrm{~min}$. Then the glass was immersed five times and air dried after every dipping in a $\left[\mathrm{Ni}(\mathrm{acac})_{2}\right]$ (ACROS) solution (MeCN $\left.0.5 \mathrm{mM}\right)$. The FTO plate was dried and a layer of NiO paste (Ni-Nanoxide N/SP, Solaronix) was screen printed (90T, Serilith AG, Switzerland). The printed plate was kept in an EtOH chamber for 3 min to reduce surface irregularities of the printed layer and dried for $6 \mathrm{~min}$ at $125{ }^{\circ} \mathrm{C}$ on a heating plate. Screen printing was used to give either one or two layers of $\mathrm{NiO}$ and then the electrodes were heated from room temperature to $350{ }^{\circ} \mathrm{C}$ over a period of $30 \mathrm{~min}$, then kept at $350{ }^{\circ} \mathrm{C}$ for $30 \mathrm{~min}$, then allowed to cool slowly to room temperature over $\sim 2 \mathrm{~h}$. After sintering, the FTO plate with the NiO screen printed dots was cut to make electrodes of size of $1 \mathrm{~cm} \times 2 \mathrm{~cm}$. After the final sintering, the thickness of a two-layer screen printed NiO surface was typically $\sim 2.5 \pm 1.0 \mu \mathrm{m}$ (by FIB measurements, recorded using a REM-FEI Helios NanoLab 650). The electrodes were heated at $250{ }^{\circ} \mathrm{C}$ for $20 \mathrm{~min}$ and then cooled to $80{ }^{\circ} \mathrm{C}$ before being immersed to a MeCN (0.3 mM) solution of P1 (Dyenamo AB) or $\left[\mathrm{Ru}(\mathrm{bpy})_{2}(5)\right](\mathrm{EtOH}, 0.1 \mathrm{mM})$ for $20 \mathrm{~h}$. The electrodes were removed from the solutions and were washed with EtOH and dried under a stream of $\mathrm{N}_{2}$. The fracture surfaces of electrodes screen printed with one or two layers of $\mathrm{NiO}$ were also examined by field-emission scanning electron microscopy (FE-SEM) using a Hitachi S-4800 equipped with a cold field-emission electron source.

Commercial counter electrodes (Solaronix Test Cell Platinum Electrodes) were washed with EtOH and then heated on a hot plate at $450{ }^{\circ} \mathrm{C}$ for $30 \mathrm{~min}$ to remove volatile organic impurities.

The DSCs were assembled by combining dye-covered FTO/ $\mathrm{NiO}$ electrodes and Pt counter-electrodes using thermoplast hot-melt sealing foil (Solaronix, Meltonix 1170-25 Series, $60 \mu \mathrm{m}$ thick) by heating while pressing them together. The electrolyte 
comprised $\mathrm{I}_{2}(0.1 \mathrm{M})$, LiI $(1 \mathrm{M})$ in MeCN. The electrolyte was introduced into the cell by vacuum backfilling. The hole on the counter electrode was finally sealed using the hot-melt sealing foil and a cover glass.

\section{Device performance measurements}

The solar cell measurements were made using duplicate cells; the active area was $0.237 \mathrm{~cm}^{2}$. The DSCs were sun soaked from behind for $20 \mathrm{~min}$ at 1 sun irradiation and then measured immediately to obtain the current density-voltage $(J-V)$ measurements with a LOT Quantum Design LS0811 instrument $\left(100 \mathrm{~mW} \mathrm{~cm}^{-2}=1\right.$ sun at AM1.5 and $23{ }^{\circ} \mathrm{C}$ ). The instrument software was set to a p-type measurement mode (inverted configuration), with $360 \mathrm{~ms}$ as the settling time, and with a voltage step of $5.3 \mathrm{mV}$. The voltage was scanned from negative to positive values.

\section{Results and discussion}

\section{Synthesis and characterization of the $\left[\operatorname{Ru}(b p y)_{2}\left(C^{\wedge} N\right)\right]^{+}$building block}

Sensitizers for n-type DSCs incorporate a wide range of anchoring units. ${ }^{25}$ In order to establish a similar palette for p-type $\left[\mathrm{Ru}(\mathrm{bpy})_{2}\left(\mathrm{C}^{\wedge} \mathrm{N}\right)\right]^{+}$dyes, we developed a modular strategy for synthesis based on Suzuki-Miyaura cross coupling and Miyaura borylation. The approach described below has the advantage that it can also easily be adapted to tune the electronic properties of the dye by replacing bpy by functionalized-bpy ligands in the cis-[Ru(bpy $\left.)_{2} \mathrm{Cl}_{2}\right]$ precursor.

Scheme 2 shows the preparation of two $\mathrm{HC}^{\wedge} \mathrm{N}$ ligands, $\mathrm{H} 1$ and $\mathrm{H} 2$, which, once incorporated into a $\left\{\mathrm{Ru}\left(\mathrm{N}^{\wedge} \mathrm{N}\right)_{2}\left(\mathrm{C}^{\wedge} \mathrm{N}\right)\right\}$-core, are capable of undergoing a Suzuki-Miyaura cross coupling. To increase the selectivity of the first step in Scheme 2, 2-bromo4-chloropyridine was used instead of 2,4-dibromopyridine. A Suzuki reaction between 2-bromo-4-chloropyridine and phenylboronic acid gave $\mathrm{H1}$ in $64 \%$ yield. Compound $\mathrm{H} 1$ has previously<smiles>CC#Cc1cccc(B(O)O)c1</smiles><smiles>CC(C)C1(C)OB(c2ccnc(-c3ccccc3)c2)OC1(C)C</smiles>

Scheme 2 Syntheses of intermediates $\mathrm{H} 1$ and $\mathrm{H}$ 2. Conditions: (i) $\mathrm{Na}_{2} \mathrm{CO}_{3}$, $\left[\mathrm{Pd}\left(\mathrm{PPh}_{3}\right)_{2} \mathrm{Cl}_{2}\right], \mathrm{THF} / \mathrm{H}_{2} \mathrm{O}, 60{ }^{\circ} \mathrm{C}, 3 \mathrm{~h}$; (ii) bis(pinacolato)diboron, KOAc, $\left[\mathrm{Pd}(\mathrm{dppf}) \mathrm{Cl}_{2}\right]$, dioxane, reflux, $72 \mathrm{~h}$.

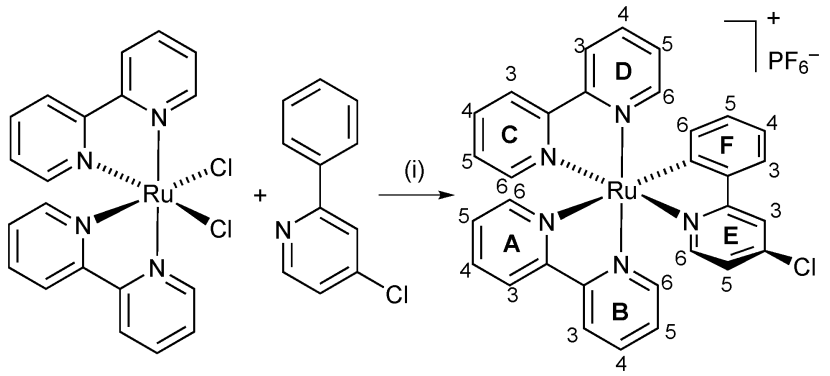

Scheme 3 Cyclometallation reaction to form $\left[\mathrm{Ru}(\mathrm{bpy})_{2}(\mathbf{1})\right]\left[\mathrm{PF}_{6}\right]$. Conditions: (i) $\mathrm{AgPF}_{6}, \mathrm{CH}_{2} \mathrm{Cl}_{2}$, reflux, $19 \mathrm{~h}$. Ring and atom labels used for NMR assignments are shown.

been prepared by a Grignard reaction with 4-chloropyridine- $N$ oxide in $74 \%$ yield, ${ }^{17}$ but we find the Suzuki coupling more convenient. To widen the scope of our modular approach to $\left\{\mathrm{Ru}\left(\mathrm{N}^{\wedge} \mathrm{N}\right)_{2}\left(\mathrm{C}^{\wedge} \mathrm{N}\right)\right\}$-functionalization, we synthesized $\mathrm{H} 2$ using a Miyaura borylation (Scheme 2). The reaction was monitored by ${ }^{1} \mathrm{H}$ NMR spectroscopy and after $\sim 5$ hours, $40 \%$ conversion had been achieved. Attempts to increase the conversion using longer reaction times and higher ratios of catalyst, base or bis(pinacolato)diboron failed. The mixture of product and reagents were subjected to a Kugelrohr distillation and the residue was chromatographed. However, pure $\mathrm{H} 2$ could not be obtained. Further development of the synthetic strategy therefore utilized ligand $\mathrm{H} \mathbf{1}$.

The reaction of $\mathrm{H} \mathbf{1}$ with cis-[Ru(bpy $\left.)_{2} \mathrm{Cl}_{2}\right]$ in the presence of $\mathrm{AgPF}_{6}\left(\right.$ Scheme 3) following a procedure for $\left[\mathrm{Ru}(\mathrm{bpy})_{2}(\mathrm{ppy})\right]^{+}$ previously reported by one of $\mathrm{us}^{26}$ gave $\left[\mathrm{Ru}(\mathrm{bpy})_{2}(\mathbf{1})\right]\left[\mathrm{PF}_{6}\right]$ in $65.2 \%$ yield. The LC-ESI mass spectrum of the product exhibits a peak envelope at $m / z 602.1$ arising from the $\left[\mathrm{M}-\mathrm{PF}_{6}\right]^{+}$ion. Fig. 2 and Fig. S1 (ESI $\dagger$ ) show the solution ${ }^{1} \mathrm{H}$ and ${ }^{13} \mathrm{C}$ NMR spectra, respectively, which were assigned using $2 \mathrm{D}$ methods (COSY, NOESY, HMQC and HMBC). The presence of the $\mathrm{C}^{\wedge} \mathrm{N}$ chelate leads to inequivalent bpy ligands, as indicated by the ring labels in Scheme 3. A starting point for ${ }^{1} \mathrm{H}$ and ${ }^{13} \mathrm{C} \mathrm{NMR}$ signal assignment is the lowest frequency signal at $\delta 6.46 \mathrm{ppm}$ in the ${ }^{1} \mathrm{H}$ NMR spectrum (Fig. 2) which is characteristic of proton $\mathrm{H}^{\mathrm{F} 6}$ of the cyclometallated phenyl ring. ${ }^{16}$

We recently commented on the paucity of structural data for $\left[\mathrm{Ru}\left(\mathrm{N}^{\wedge} \mathrm{N}\right)_{2}\left(\mathrm{C}^{\wedge} \mathrm{N}\right)\right]^{+}$complexes. ${ }^{16}$ An updated search of the Cambridge Structural Database (CSD v. 5.37 with one update) using Conquest v. 1.18 revealed only 25 hits for discrete

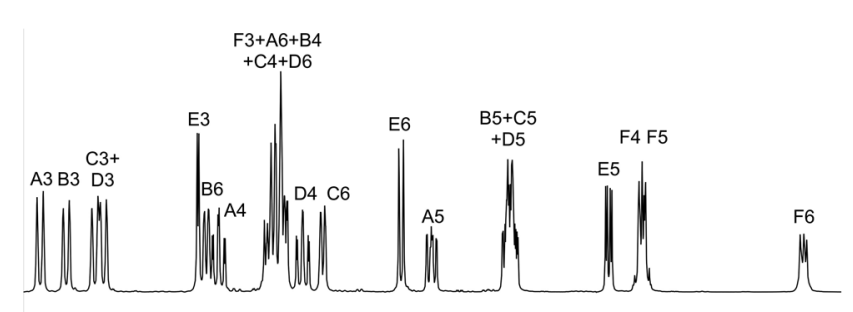

$\begin{array}{llllllllllllllllllllll}3.5 & 8.4 & 8.3 & 8.2 & 8.1 & 8.0 & 7.9 & 7.8 & 7.7 & 7.6 & 7.5 & 7.4 & 7.3 & 7.2 & 7.1 & 7.0 & 6.9 & 6.8 & 6.7 & 6.6 & 6.5 & 6.4\end{array}$ Fig. 2 The $500 \mathrm{MHz}{ }^{1} \mathrm{H}$ NMR spectrum of $\left[R u(b p y)_{2}(\mathbf{1})\right]\left[\mathrm{PF}_{6}\right]$ (in $\mathrm{CD}_{3} \mathrm{CN}$ ). Atom labels are given in Scheme 3. 


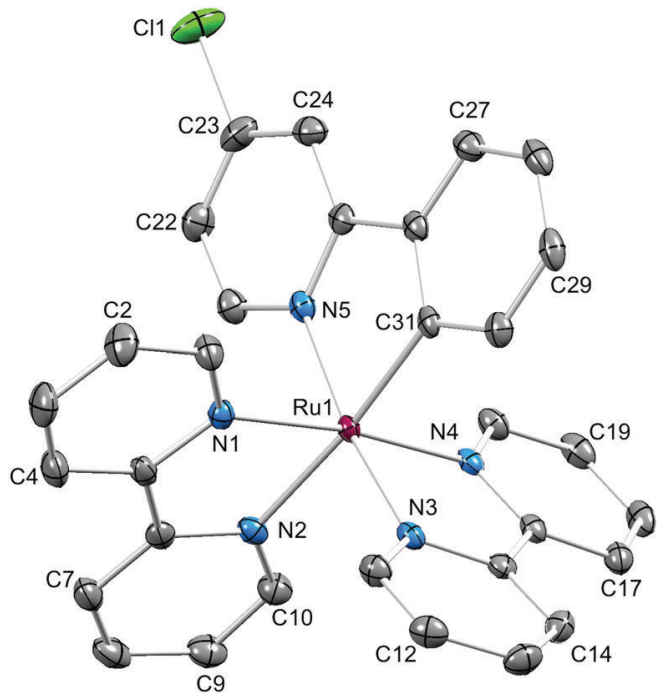

Fig. 3 Structure of the $\Lambda$-[Ru(bpy $\left.)_{2}(\mathbf{1})\right]^{+}$cation in $\left[R u(b p y)_{2}(\mathbf{1})\right]\left[\mathrm{PF}_{6}\right]$ with ellipsoids plotted at $40 \%$ probability level and $\mathrm{H}$ atoms omitted for clarity. Selected bond parameters: Ru1-N1 $=2.0591(17)$, Ru1-N2 $=2.1378(19)$, Ru1-N3 = 2.0360(18), Ru1-N4 = 2.0400(18), Ru1-N5 = 2.0742(18), Ru1-C31 = 2.038(2), C23-Cl1 = 1.735(2) $\AA ; \mathrm{N} 1-\mathrm{Ru} 1-\mathrm{N} 2=77.67(7)$, N3-Ru1-N4 = 79.11(7), N5-Ru1-C31 = 79.76(8), N1-Ru1-N4 = 172.76(7), N3-Ru1-N5 = 171.53(7), N2-Ru1-C31 = 174.17(8) .

complexes containing a $\left\{\mathrm{Ru}(\mathrm{bpy})_{2}(\mathrm{ppy})\right\}$-core (Hppy $=2$-phenylpyridine; the bpy core-unit includes complexes with phen ligands). Single crystals of $\left[\mathrm{Ru}(\mathrm{bpy})_{2}(\mathbf{1})\right]\left[\mathrm{PF}_{6}\right]$ were grown by vapour diffusion of $\mathrm{Et}_{2} \mathrm{O}$ into an MeCN solution of the complex. The compound crystallizes in the monoclinic space group $P 2_{1} / n$ with both the $\Lambda$ - and $\Delta$-[Ru(bpy $\left.)_{2}(\mathbf{1})\right]^{+}$cations present in the unit cell. Fig. 3 shows the structure of the $\Lambda$-[Ru(bpy $\left.)_{2}(\mathbf{1})\right]^{+}$ cation and selected bond distances and angles are given in the figure caption. The structure exhibits no surprises, being similar to that of the analogous complex in which the chloro substituent in coordinated [1] $]^{-}$is replaced by a methyl acetate group. ${ }^{16}$ As in the latter structure, efficient face-to-face and edge-to-face $\pi$-contacts between enantiomers is observed (Fig. 4). For the face-to-face interaction, the distance between the ring planes $=3.31 \AA$, and centroid $\cdots$ centroid separation = $3.64 \AA$; for the edge-to-face interaction, $\mathrm{C}-\mathrm{H} \cdots$ centroid distance $=2.48 \AA$.

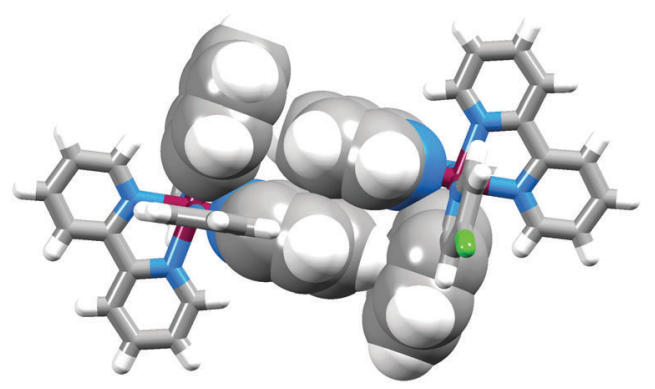

Fig. 4 Face-to-face and edge-to-face contacts between $\Lambda^{-}$and $\Delta-\left[\mathrm{Ru}(\mathrm{bpy})_{2}(\mathbf{1})\right]^{+}$cations in $\left[\mathrm{Ru}(\mathrm{bpy})_{2}(\mathbf{1})\right]\left[\mathrm{PF}_{6}\right]$.

\section{Synthesis and characterization of anchoring unit building blocks}

We decided to target $\left[\mathrm{Ru}(\mathrm{bpy})_{2}\left(\mathrm{C}^{\wedge} \mathrm{N}\right)\right]^{+}$dyes with $\mathrm{CO}_{2} \mathrm{H}$ or $\mathrm{P}(\mathrm{O})(\mathrm{OH})_{2}$ anchoring groups, and therefore required anchoring modules capable of undergoing Suzuki-Miyaura cross coupling with $\left[\mathrm{Ru}(\mathrm{bpy})_{2}(\mathbf{1})\right]^{+}$. For the carboxylic acid anchor, a suitable reagent is a commercially available 4-carboxyphenylboronic acid. The phosphonic acid anchoring module was synthesized by the route shown in Scheme 4. Diethyl 4-bromobenzenephosphonate (Scheme 4) has previously been prepared in $22 \%$ from 1,4-dibromobenzene by Grignard reaction. ${ }^{18}$ We were able to isolate the phosphonate ester in $73 \%$ yield by a palladiumcatalysed phosphonation of 1-bromo-4-iodobenzene (selective at the iodo-functionality) using a stoichiometric amount of $\mathrm{HPO}(\mathrm{OEt})_{2}$. Further functionalization by Miyaura borylation using bis(pinacolato)diboron yielded the diester 3 (Scheme 4); this was used in the next step without purification. Compound 3 was recently reported as part of a wide-ranging study of regioselective aromatic $\mathrm{C}-\mathrm{H}$ borylations, ${ }^{19}$ and the ${ }^{1} \mathrm{H}$ NMR spectroscopic data agreed with those published. In the ${ }^{31} \mathrm{P}\left\{{ }^{1} \mathrm{H}\right\}$ NMR spectrum, 3 is characterized by a signal at $\delta+18.6 \mathrm{ppm}$, and in the ${ }^{11} \mathrm{~B}$ NMR spectrum by a resonance at $\delta+22.4 \mathrm{ppm}$. Deprotection of 3 to give acid $\mathrm{H}_{2} 4$ was achieved with $\mathrm{Me}_{3} \mathrm{SiBr}$. Whereas the diester 3 is readily soluble in $\mathrm{CH}_{2} \mathrm{Cl}_{2}$ and $\mathrm{CHCl}_{3}$, acid $\mathrm{H}_{2} 4$ is soluble only in solvents such as DMSO; in DMSO- $d_{6}$, $\mathrm{H}_{2} 4$ exhibits signals in the ${ }^{31} \mathrm{P}\left\{{ }^{1} \mathrm{H}\right\}$ and ${ }^{11} \mathrm{~B}$ NMR spectra at $\delta$ +12.2 and $+28.4 \mathrm{ppm}$, respectively. In the LC-ESI mass spectrum of $\mathrm{H}_{2} 4$, a peak at $m / z=285.1$ was assigned to $[\mathrm{M}+\mathrm{H}]^{+}$.

\section{Conversion of $\left[\mathrm{Ru}(\mathrm{bpy})_{2}(1)\right]^{+}$to potential sensitizers}

The final step of the synthesis of cyclometallated ruthenium complexes functionalized with anchoring groups is a cross coupling of $\left[\mathrm{Ru}(\mathrm{bpy})_{2}(\mathbf{1})\right]^{+}$with 4-carboxyphenylboronic acid or $\mathrm{H}_{2} 4$ (Scheme 5). Table 1 summarizes the reaction conditions investigated during optimization; reactions were monitored using LC-ESI-MS. Initial attempts to couple $\left[\mathrm{Ru}(\mathrm{bpy})_{2}(\mathbf{1})\right]^{+}$with 4-carboxyphenylboronic acid using typical Suzuki coupling conditions with catalytic amounts of $\operatorname{Pd}(\mathrm{OAc})_{2}$ did not give the desired product. Although $\left[\mathrm{Ru}(\mathrm{bpy})_{2}(\mathbf{1})\right]^{+}$was consumed, LC-ESI-MS confirmed that the products were $\left[\mathrm{Ru}(\mathrm{bpy})_{2}(\mathrm{ppy})\right]^{+}$(resulting from palladiumcatalysed dehalogenation) and the homocoupled product

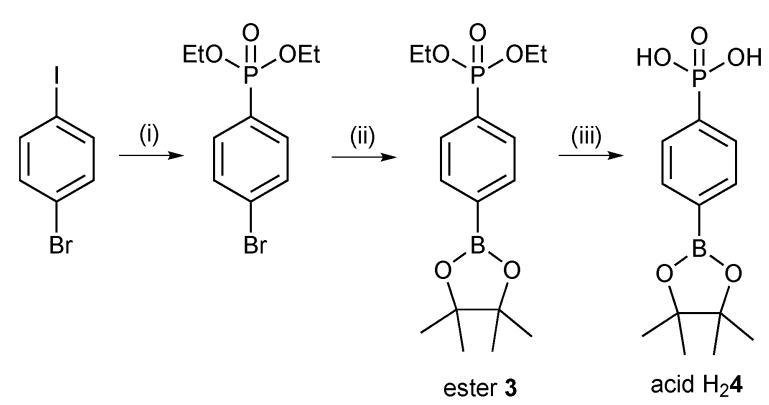

Scheme 4 Synthesis of the phosphonic acid building block 4 . Conditions: (i) $\mathrm{HPO}(\mathrm{OEt})_{2}, \mathrm{Cs}_{2} \mathrm{CO}_{3},\left[\mathrm{Pd}\left(\mathrm{PPh}_{3}\right)_{4}\right], \mathrm{THF}$, reflux, $2.5 \mathrm{~h}$; (ii) bis(pinacolato)diboron, KOAC, $\left[\mathrm{Pd}(\mathrm{dppf}) \mathrm{Cl}_{2}\right], \mathrm{THF}$, microwave reactor, $90{ }^{\circ} \mathrm{C}, 1 \mathrm{~h}$; (iii) $\mathrm{Me}_{3} \mathrm{SiBr}, \mathrm{CH}_{2} \mathrm{Cl}_{2}$, room temperature, $19 \mathrm{~h}$. 


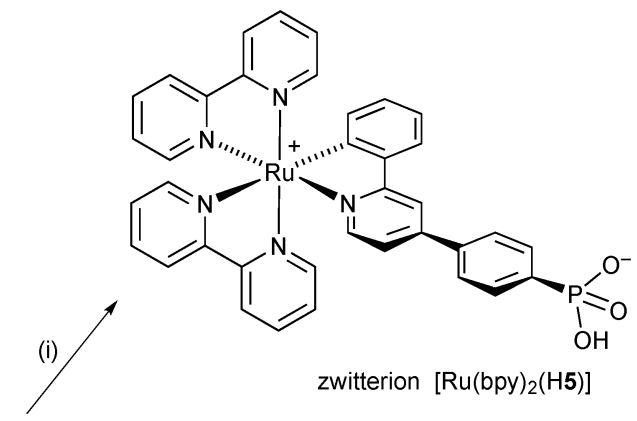

$\left[\mathrm{Ru}(\mathrm{bpy})_{2}(\mathbf{1})\right]\left[\mathrm{PF}_{6}\right]$

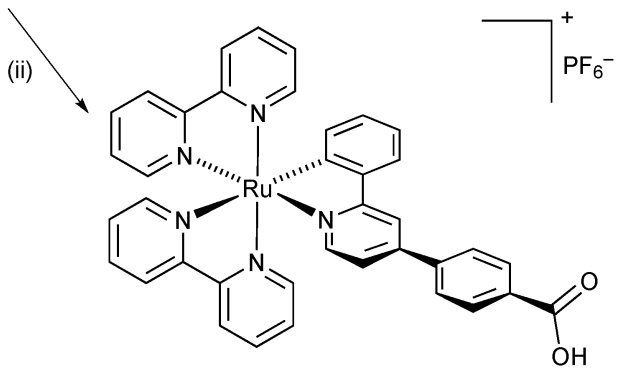

$\left[\mathrm{Ru}(\mathrm{bpy})_{2}(\mathrm{H} 6)\right][\mathrm{PF} 6]$

Scheme 5 Conversion of $\left[\mathrm{Ru}(\mathrm{bpy})_{2}(\mathbf{1})\right]\left[\mathrm{PF} \mathrm{F}_{6}\right]$ to $\left[\mathrm{Ru}(\mathrm{bpy})_{2}(\mathrm{H} 5)\right]$ and $\left[\mathrm{Ru}(\mathrm{bpy})_{2}-\right.$ (H6)] $\left[\mathrm{PF}_{6}\right]$. Optimized conditions: (i) $\mathrm{H}_{2} 4, \mathrm{~K}_{3} \mathrm{PO}_{4}$, SPhos Pd G2, MeCN/ $/ \mathrm{H}_{2} \mathrm{O}$; $70{ }^{\circ} \mathrm{C}, 5 \mathrm{~h}$; (ii) 4-carboxyphenylboronic acid, $\mathrm{K}_{3} \mathrm{PO}_{4}$, SPhos Pd G2, MeCN/ $\mathrm{H}_{2} \mathrm{O} ; 70{ }^{\circ} \mathrm{C}, 5 \mathrm{~h}$.

$\left[(\text { bpy })_{2} \operatorname{Ru}(\mu \text {-dppy }) \operatorname{Ru}(\text { bpy })_{2}\right]^{+}$(dppy $=2,2^{\prime}$-diphenyl-4,4'-bipyridine $)$. To overcome this problem, we turned to a catalyst containing the sterically demanding SPhos ligand which has been shown by $\mathrm{O}^{\prime} \mathrm{Connor}^{27}$ to be effective for the Suzuki coupling of 4-carboxyphenylboronic acid with an aryl chloride. The second generation precatalyst SPhos Pd G2 does not require reducing agents for activation and is highly reactive. Under microwave conditions (Table 1), Suzuki-Miyaura coupling of $\left[\mathrm{Ru}(\mathrm{bpy})_{2}(\mathbf{1})\right]^{+}$with 4-carboxyphenylboronic acid using SPhos Pd G2 in EtOH solvent showed a 91\% selectivity for the desired product and no homocoupling was observed. However, if similar conditions are used for the reaction between $\left[\mathrm{Ru}(\mathrm{bpy})_{2}(\mathbf{1})\right]^{+}$and $\mathrm{H}_{2} \mathbf{4}$, only $31 \%$ selectivity was achieved (Table 1). The choice of solvent is known to influence the dehalogenation reaction, ${ }^{28}$ and therefore EtOH

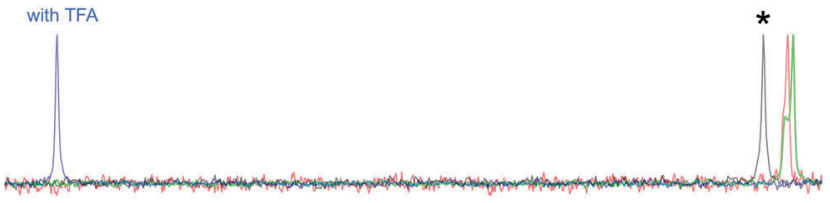

$\begin{array}{llllllllllll}15.2 & 14.8 & 14.4 & 14.0 & 13.6 & 13.2 & 12.8 & 12.4 & 12.0 & 11.6 & 11.2 & 10.8\end{array}$

Fig. $5162 \mathrm{MHz}{ }^{31} \mathrm{P}\left\{{ }^{1} \mathrm{H}\right\}$ NMR spectrum of $\left[\mathrm{Ru}(\mathrm{bpy})_{2}(\mathrm{H} 5)\right]{ }^{(*)}$ in $\mathrm{CD}_{3} \mathrm{OD}$, and spectra taken after the addition of (a) $\mathrm{CF}_{3} \mathrm{CO}_{2} \mathrm{H}$ (TFA, blue), (b) $\mathrm{K}_{2} \mathrm{CO}_{3}$ (green) or (c) $\mathrm{CF}_{3} \mathrm{CO}_{2} \mathrm{H}$ followed by $\mathrm{K}_{2} \mathrm{CO}_{3}$ (red).

was replaced by a 1:1 mixture of MeCN and $\mathrm{H}_{2} \mathrm{O}$. This choice was made, in part, for solubility reasons. Under the conditions shown in Table 1, the Suzuki-Miyaura cross coupling of $\left[\mathrm{Ru}(\mathrm{bpy})_{2}(\mathbf{1})\right]^{+}$and 4-carboxyphenylboronic acid proceeded with both $100 \%$ conversion and selectivity. For the reaction with anchoring module $\mathrm{H}_{2} 4$, the highest selectivity achieved was 97\%; adjustments to the temperature and time (Table 1) were required for optimization of selectivity.

The electrospray mass spectra of the products revealed peak envelopes at $m / z 724.2$ and 688.1, respectively. However, on their own, these results are ambiguous, because they are consistent with either $[\mathrm{M}+\mathrm{H}]^{+}$for $\mathrm{M}$ being a zwitterion $\left[\mathrm{Ru}(\mathrm{bpy})_{2}(\mathrm{H} 5)\right]$ or $\left[\mathrm{Ru}(\mathrm{bpy})_{2}(\mathbf{6})\right]$, or $\left[\mathrm{M}-\mathrm{PF}_{6}\right]^{-}$for salts $\left[\mathrm{Ru}(\mathrm{bpy})_{2}\left(\mathrm{H}_{2} 5\right)\right]\left[\mathrm{PF}_{6}\right]$ and $\left[\mathrm{Ru}(\mathrm{bpy})_{2}(\mathrm{H} 6)\right]\left[\mathrm{PF}_{6}\right]$. Elemental analysis for the phosphonic acid-functionalized compound was consistent with the zwitterion shown in Scheme 5, and this was further supported by the response of the ${ }^{31} \mathrm{P}$ NMR spectrum to the addition of base or acid. Fig. 5 shows that the signal at $\delta+10.8 \mathrm{ppm}$ that characterizes the isolated complex shifts to $\delta+15.2 \mathrm{ppm}$ after TFA vapour has been blown over the mouth of the NMR tube. Addition of a little solid $\mathrm{K}_{2} \mathrm{CO}_{3}$ to the same NMR sample results in a shift back to lower frequency $(\delta+10.6 \mathrm{ppm}$ with a shoulder at $\delta+10.7 \mathrm{ppm})$. A resonance at approximately the same frequency results if $\mathrm{K}_{2} \mathrm{CO}_{3}$ is added to a solution of the isolated complex. These observations indicate that the complex is the zwitterion $\left[\mathrm{Ru}(\mathrm{bpy})_{2}(\mathrm{H} 5)\right]$. In contrast, the second product is formulated as $\left[\mathrm{Ru}(\mathrm{bpy})_{2}(\mathrm{H} 6)\right]\left[\mathrm{PF}_{6}\right]$; the ${ }^{31} \mathrm{P}$ NMR spectrum showed a septet at $\delta-144.7 \mathrm{ppm}\left(J_{\mathrm{PF}}=727 \mathrm{~Hz}\right)$ characteristic of the hexafluoridophosphate anion. The difference in protonation states in the isolated ruthenium complexes is consistent

Table 1 Conditions and product selectivity for Suzuki-Miyaura cross coupling of $\left[\mathrm{Ru}(\mathrm{bpy})_{2}(\mathbf{1})\right]^{+}$with 4 -carboxyphenylboronic acid or $\mathrm{H}_{2} \mathbf{4}$

\begin{tabular}{|c|c|c|c|c|c|}
\hline Catalyst/precatalyst & $t / \mathrm{h}$ & $\mathrm{Temp} /{ }^{\circ} \mathrm{C}$ & Solvent & Conversion $^{a} / \%$ & Selectivity C : Dehal $: \mathrm{H}^{a, b}$ \\
\hline $\mathrm{Pd}(\mathrm{OAc})_{2}$ & 24 & $\sim 22$ & $\mathrm{THF} / \mathrm{H}_{2} \mathrm{O}(4: 1 \mathrm{vol})$ & 100 & $0: 59: 41$ \\
\hline \multicolumn{6}{|l|}{ Coupling with $\mathrm{H}_{2} 4$} \\
\hline SPhos Pd G2 & 0.5 & 80 & $\mathrm{EtOH}^{c}$ & 75 & $31: 69: 0$ \\
\hline SPhos Pd G2 & 1 & 90 & $\mathrm{MeCN} / \mathrm{H}_{2} \mathrm{O}(1: 1 \mathrm{vol})$ & 100 & $90: 7: 3$ \\
\hline
\end{tabular}

${ }^{a} 100 \%$ conversion corresponds to all $\left[\mathrm{Ru}(\mathrm{bpy})_{2}(\mathbf{1})\right]^{+}$consumed; values are calculated from LC-ESI-MS peak integration assuming that all measured complexes have an equal response factor in the ESI-MS. ${ }^{b} \mathrm{C}=$ coupled product $\left[\mathrm{Ru}(\mathrm{bpy})_{2}(\mathrm{H} 5)\right]$ or $\left[\mathrm{Ru}(\mathrm{bpy})_{2}(\mathrm{HG})\right]\left[\mathrm{PF}_{6}\right] ; \mathrm{Dehal}=\left[\mathrm{Ru}^{\mathrm{b}}(\mathrm{bpy})_{2}(\mathrm{Ppy})\right]\left[\mathrm{PF}_{6}\right]$; $\mathrm{H}=$ homocoupled product $\left[(\mathrm{bpy})_{2} \mathrm{Ru}(\mu\right.$-dppy $\left.) \mathrm{Ru}(\mathrm{bpy})_{2}\right]\left[\mathrm{PF}_{6}\right]_{2} \cdot{ }^{c}$ Reaction performed under microwave conditions. 
with the $\mathrm{p} K_{\mathrm{a}}$ values of structurally related pairs of carboxylic and phosphonic acids, e.g. for $\mathrm{PhCO}_{2} \mathrm{H}, \mathrm{p} K_{\mathrm{a}}=4.20$ and for $\mathrm{PhPO}_{3} \mathrm{H}_{2}, \mathrm{p} K_{\mathrm{a}}(1)=1.86 .^{29}$

The complexes $\left[\mathrm{Ru}(\mathrm{bpy})_{2}(\mathrm{H} 5)\right]$ and $\left[\mathrm{Ru}(\mathrm{bpy})_{2}(\mathrm{H} 6)\right]\left[\mathrm{PF}_{6}\right]$ were characterized by ${ }^{1} \mathrm{H}$ and ${ }^{13} \mathrm{C}$ NMR spectroscopies, the spectra being assigned using COSY, HMQC and HMBC methods. The ${ }^{1} \mathrm{H}$ NMR spectra are shown in Fig. S2 (ESI $\dagger$ ). We noted that when an acetone- $d_{6}$ solution of $\left[\mathrm{Ru}(\mathrm{bpy})_{2}(\mathrm{H} 6)\right]\left[\mathrm{PF}_{6}\right]$ was left to stand for several days, the ${ }^{1} \mathrm{H}$ NMR signals for protons $\mathrm{H}^{\mathrm{G} 2}$ and $\mathrm{H}^{\mathrm{G} 3}$ (the ring to which the $\mathrm{CO}_{2} \mathrm{H}$ group is attached) broadened and shifted (Fig. S3, ESI $\dagger$ ); some precipitate was also observed in the NMR tube. We attribute this to a change in protonation state, but have not investigated the system in detail.

The solution absorption spectra of $\left[\mathrm{Ru}(\mathrm{bpy})_{2}(\mathrm{H} 5)\right]$ and $\left[\mathrm{Ru}(\mathrm{bpy})_{2}(\mathrm{H} 6)\right]\left[\mathrm{PF}_{6}\right]$ are similar, and the features reflect those of $\left[\mathrm{Ru}(\mathrm{bpy})_{2}\left(\mathrm{C}^{\wedge} \mathrm{N}\right)\right]^{+}$complexes previously described. . $^{71,16,30,31}$ The spectrum of $\left[\mathrm{Ru}(\mathrm{bpy})_{2}(\mathrm{H} 5)\right]$ is shown in Fig. 6 . The intense, high-energy bands (between $\sim 240$ and $320 \mathrm{~nm}$ ) are assigned to ligand-centred $\pi^{*} \leftarrow \pi$ transitions, while the broad, lower intensity absorptions around 350-430 $\mathrm{nm}$ and $430-620 \mathrm{~nm}$ are characteristic of $\left[\mathrm{Ru}(\mathrm{bpy})_{2}\left(\mathrm{C}^{\wedge} \mathrm{N}\right)\right]^{+}$cations and arise, respectively, from metal-to- $\mathrm{C}^{\wedge} \mathrm{N}$ and metal-to-bpy MLCT transitions. ${ }^{7,11,31}$ The broad response of $\left[\mathrm{Ru}(\mathrm{bpy})_{2}(\mathrm{H} 5)\right]$ is comparable to that of the organic dye P1 (Scheme 6 and Fig. 6) that is a standard sensitizer in p-type DSCs (see later).

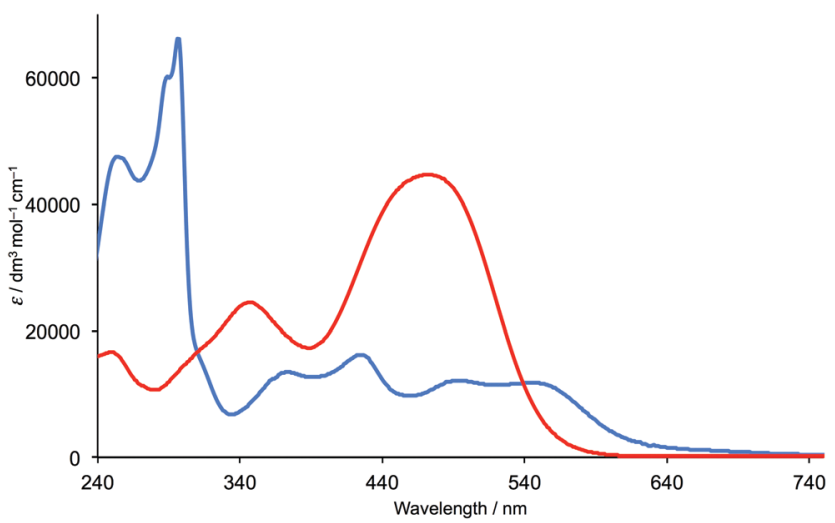

Fig. 6 The solution absorption spectra of $\left[\mathrm{Ru}(\mathrm{bpy})_{2}(\mathrm{H} 5)\right](\mathrm{MeOH}$, $1 \times 10^{-5} \mathrm{~mol} \mathrm{dm}^{-3}$, blue curve) and of the dye P1 (MeCN, $1 \times$ $10^{-5} \mathrm{~mol} \mathrm{dm}^{-3}$, red curve).

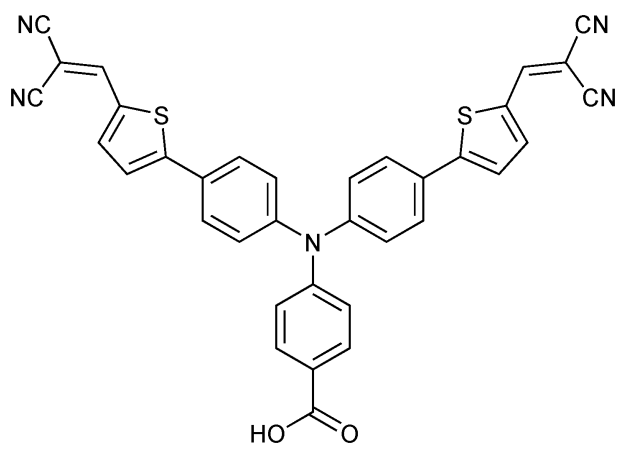

Scheme 6 Structure of sensitizer P1.
Table 2 Redox potentials for $\left[\mathrm{Ru}(\mathrm{bpy})_{2}(\mathrm{H} 5)\right]$ (degassed DMSO solutions) and $\left[\mathrm{Ru}(\mathrm{bpy})_{2}(\mathrm{H} 6)\right]\left[\mathrm{PF}_{6}\right]$ compared to $\left[\mathrm{Ru}(\mathrm{bpy})_{2}(\mathrm{ppy})\right]\left[\mathrm{PF}_{6}\right]^{16}$ (both in degassed $\mathrm{MeCN}$ ) with respect to $\mathrm{Fc} / \mathrm{Fc}^{+} ; 0.1 \mathrm{M}\left[{ }^{n} \mathrm{Bu}_{4} \mathrm{~N}\right]\left[\mathrm{PF}_{6}\right]$ as supporting electrolyte and a scan rate of $0.1 \mathrm{~V} \mathrm{~s}^{-1}$

\begin{tabular}{lccccc}
\hline Complex & $E_{1 / 2}^{\mathrm{ox}} / \mathrm{V}$ & $E_{1 / 2}^{\mathrm{red} 1} / \mathrm{V}$ & $E_{1 / 2}^{\mathrm{red} 2} / \mathrm{V}$ & $E_{1 / 2}^{\mathrm{red} 3} / \mathrm{V}$ & $\Delta E_{1 / 2} / \mathrm{V}$ \\
\hline$\left[\mathrm{Ru}(\mathrm{bpy})_{2}(\mathrm{ppy})\right]\left[\mathrm{PF}_{6}\right]$ & +0.09 & -1.96 & -2.22 & & 2.05 \\
{$\left[\mathrm{Ru}(\mathrm{bpy})_{2}(\mathrm{H} 5)\right]$} & +0.05 & -1.96 & -2.23 & -2.83 & 2.01 \\
{$\left[\mathrm{Ru}(\mathrm{bpy})_{2}(\mathrm{H} 6)\right]\left[\mathrm{PF}_{6}\right]$} & +0.07 & -1.94 & -2.21 & -2.82 & 2.02
\end{tabular}

The cyclometallated ruthenium(II) complexes are redox active and cyclic voltammetric data compared to the archetype compound $\left[\mathrm{Ru}(\mathrm{bpy})_{2}(\mathrm{ppy})\right]\left[\mathrm{PF}_{6}\right]$ are given in Table 2 .

The modular approach to the synthesis of $\left[\mathrm{Ru}(\mathrm{bpy})_{2}(\mathrm{H} 5)\right]$ and $\left[\mathrm{Ru}(\mathrm{bpy})_{2}(\mathrm{H6})\right]\left[\mathrm{PF}_{6}\right]$ is a versatile one that should be applicable for introducing other anchoring domains. [Ru(bpy $\left.)_{2}(\mathrm{H} 5)\right]$ is more reliably isolated in an unambiguous protonation state than $\left[\mathrm{Ru}(\mathrm{bpy})_{2}(\mathrm{H} 6)\right]\left[\mathrm{PF}_{6}\right]$ (see above) and, therefore, we chose to focus on the use of $\left[\mathrm{Ru}(\mathrm{bpy})_{2}(\mathrm{H} 5)\right]$ as a sensitizer in p-type DSCs in the present investigation.

\section{DSC working-electrode fabrication}

The fabrication of $\mathrm{FTO} / \mathrm{NiO}$ photocathodes in p-type DSCs is a critical part of the device fabrication. ${ }^{32,33}$ Initially, ${ }^{34}$ we investigated doctor blading and screen-printing the FTO glass with different numbers of layers of $\mathrm{NiO}$, combined with pre-treatment of the FTO glass with dip-coated or spin-coated $\left[\mathrm{Ni}(\mathrm{OAc})_{2}\right]$ or $\left[\mathrm{Ni}(\mathrm{acac})_{2}\right]$ to improve adhesion of the NiO paste. ${ }^{35,36}$ The surface morphology of the electrodes was investigated using SEM and FIB imaging (see Experimental section). Pretreating the FTO glass with $\left[\mathrm{Ni}(\mathrm{acac})_{2}\right]$ followed by two screen-printed layers of NiO paste lead (after sintering involving a cycle between room temperature and $350{ }^{\circ} \mathrm{C}$, see Experimental section) to a NiO layer thickness of $\sim 2.5 \pm 1.0 \mu \mathrm{m}$ (Fig. 7). This is typical of $\mathrm{NiO}$ photocathodes used in p-type DSC studies ${ }^{37,38}$ and is compatible with the limitation imposed by the diffusion length of a hole in the NiO semiconductor. ${ }^{38}$ A recent investigation using the standard P1 dye (Scheme 6) ${ }^{33}$ confirms that two-layers of screen-printed commercial (Dyenamo) NiO lead to better performing p-type DSCs than using one layer.

The dye-functionalized photocathodes were prepared by immersing the FTO/NiO electrodes in an MeCN solution of P1 (Scheme 6) or an EtOH solution of $\left[\mathrm{Ru}(\mathrm{bpy})_{2}(\mathrm{H} 5)\right]$. The solid-state absorption spectra of electrodes with adsorbed dye are shown in Fig. 8. Each spectrum was corrected for the background spectrum of the blank FTO/NiO electrode. A comparison of Fig. 6 and 8 shows that the profiles of the absorption spectra are similar, although there is a red-shift on going from solution to solid; for P1, $\lambda_{\max }=468 \mathrm{~nm}$ in $\mathrm{MeCN}$ and $\sim 525 \mathrm{~nm}$ in the solid-state dye, and for $\left[\mathrm{Ru}(\mathrm{bpy})_{2}(\mathrm{H} 5)\right], \lambda_{\max }=492$ and $544 \mathrm{~nm}$ in $\mathrm{MeOH}$, compared to $\sim 500$ and $\sim 560 \mathrm{~nm}$ in the solid sample.

\section{DSC performances}

The p-type DSCs were assembled as described in the Experimental Section, and duplicate DSCs were made for each dye. Previous investigations of cyclometallated ruthenium(II) dyes 


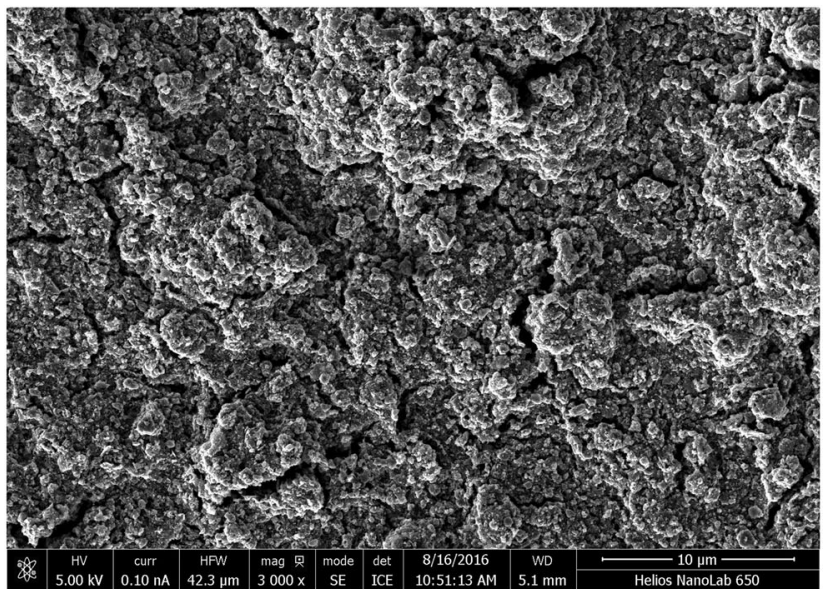

(a)

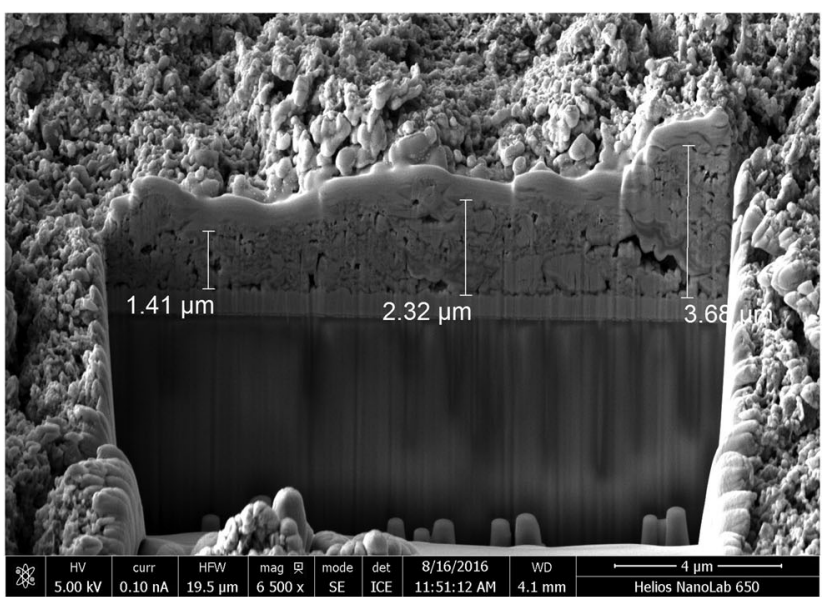

(b)

Fig. 7 FIB images of an $\mathrm{FTO} / \mathrm{NiO}$ electrode with [Ni(acac $)_{2}$ ] pretreatment and two screen-printed layers of $\mathrm{NiO}$ sintered at $350{ }^{\circ} \mathrm{C}$ : (a) top surface, and (b) a gallium beam cut into the $\mathrm{NiO}$ with a platinum layer is deposited on top of the $\mathrm{NiO}$ surface. In (b), the glass and FTO coating are visible beneath the $\mathrm{NiO}$.

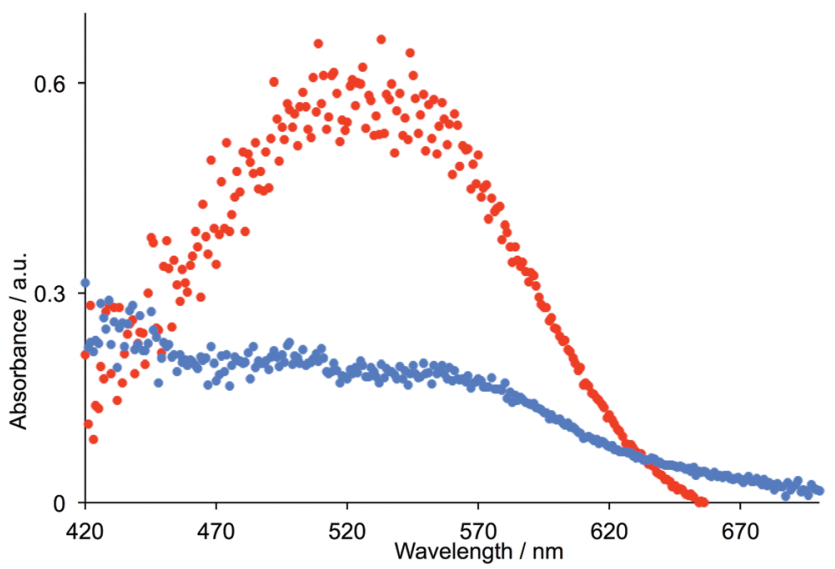

Fig. 8 Solid-state absorption spectra of FTO/NiO electrodes with adsorbed dyes $\mathrm{P} 1$ (red) and $\left[\mathrm{Ru}(\mathrm{bpy})_{2}(\mathrm{H} 5)\right]$ (blue).

have employed an electrolyte comprising $\mathrm{I}^{-} / \mathrm{I}_{3}{ }^{-} / \mathrm{MeCN}$ (with no additives), ${ }^{9-11,13}$ a composition regularly used for the standard dye P1. For DSC measurements, a settling time of $360 \mathrm{~ms}$ gave reproducible $J-V$ curves whether the voltage was scanned from negative to positive, or from positive to negative, potentials. Settling times of $\leq 200 \mathrm{~ms}$ led to $J-V$ curves that differed with the direction of the scan; a settling time of $360 \mathrm{~ms}$ was therefore adopted as standard for all measurements.

Before discussing the results, we draw attention to the fact that literature photoconversion efficiencies $(\eta)$ of standard dye P1 in p-type DSCs show significant variation. ${ }^{33,39,40}$ Contributing factors include the method of $\mathrm{NiO}$ fabrication and layer thickness, ${ }^{32,33}$ and the electrolyte $\left(\mathrm{MeCN} / \mathrm{I}_{2} / \mathrm{LiI}, \mathrm{MeCN} / \mathrm{I}_{2} /\right.$ $\mathrm{LiI} / \mathrm{TBP}$, or LiI/I $/$ / propylene carbonate). ${ }^{41-46}$ The use of MeCN in place of propylene carbonate in the electrolyte is beneficial in terms of short-circuit current density $\left(J_{\mathrm{SC}}\right)$. It is also important to note that for n-type DSCs, the use of different sun simulators (e.g. Solaronix $v s$. LOT) also leads to differing $J_{\mathrm{SC}}$ values. ${ }^{47}$ In a bench-marking investigation, ${ }^{33}$ Gibson and coworkers included measurements of the performance of p-type DCSs with P1 with a variety of different fabrication methods. The study included electrodes with one screen-printed layer of Solaronix NiO paste and a sintering temperature of $350{ }^{\circ} \mathrm{C}$, leading to values of $J_{\mathrm{SC}}=1.57 \mathrm{~mA} \mathrm{~cm}{ }^{-2}$, open-circuit voltage $\left(V_{\mathrm{OC}}\right)=93 \mathrm{mV}$, fillfactor (ff) $=32 \%$, and $\eta=0.047 \%$. We have fabricated DSCs that are directly comparable to the latter except for the inclusion of the $\mathrm{Ni}(\mathrm{acac})_{2}$ pretreatment (see above) which we find essential for good adhesion of the NiO to the FTO glass. The performance data for P1 (Table 3, one-layer of NiO) are similar to those reported, ${ }^{33}$ validating the data presented below. We find an enhanced DSC performance for P1 is obtained by using twolayers of NiO (Table 3).

Table 3 also gives values of $J_{\mathrm{SC}}, V_{\mathrm{OC}}$, ff and $\eta$ for DSCs sensitized with the cyclometallated dye $\left[\mathrm{Ru}(\mathrm{bpy})_{2}(\mathrm{H} 5)\right]$ with electrodes made with one or two screen-printed layers of NiO. As for P1, better DSC performances are observed for two-layers of NiO. $J-V$ curves for the DSCs containing [Ru(bpy $\left.)_{2}(\mathrm{H} 5)\right]$ adsorbed on two-layers of $\mathrm{NiO}$ are shown in Fig. 9. The low fill-factors of p-type DSCs are a known phenomenon. ${ }^{48}$ Pairs of DSCs (Table 3 and Fig. 9) give reproducible DSC parameters. Pleasingly, $J_{\mathrm{SC}}$ values and $\eta$ are significantly better for $\left[\mathrm{Ru}(\mathrm{bpy})_{2}(\mathrm{H} 5)\right]$ than for $\mathrm{P} 1$, and both $J_{\mathrm{SC}}$ and $\eta$ are comparable with the best values $\left(J_{\mathrm{SC}}=3.43 \mathrm{~mA} \mathrm{~cm}^{-2} \text { and } \eta=0.109 \% \text { for the dye shown in Scheme } 7\right)^{9}$ reported for cyclometallated ruthenium dyes ${ }^{9-11,13}$ or for two recently reported diacetylide ruthenium(II) donor- $\pi$-acceptor

Table 3 Performance data for duplicate DSCs containing dyes $\left[R u(b p y)_{2}(\mathbf{5})\right]$ or P1. Measurements were made on the day of sealing the DSCs

\begin{tabular}{|c|c|c|c|c|c|}
\hline Dye (DSC number) & $\begin{array}{l}\text { Number of } \\
\text { NiO layers }\end{array}$ & $J_{\mathrm{SC}} / \mathrm{mA} \mathrm{cm}^{-2}$ & $V_{\mathrm{OC}} / \mathrm{mV}$ & $\mathrm{ff} / \%$ & $\eta / \%$ \\
\hline P1 (cell 1) & 1 & 1.54 & 91 & 35 & 0.049 \\
\hline P1 (cell 2) & 1 & 1.26 & 95 & 35 & 0.042 \\
\hline P1 (cell 1) & 2 & 1.84 & 88 & 35 & 0.057 \\
\hline P1 (cell 2) & 2 & 1.96 & 82 & 32 & 0.051 \\
\hline$\left[\mathrm{Ru}(\mathrm{bpy})_{2}(\mathrm{H} 5)\right]$ (cell 1) & 1 & 2.18 & 93 & 39 & 0.079 \\
\hline$\left[\mathrm{Ru}(\mathrm{bpy})_{2}(\mathrm{H} 5)\right]$ (cell 2) & 1 & 2.00 & 94 & 41 & 0.077 \\
\hline$\left[\mathrm{Ru}(\mathrm{bpy})_{2}(\mathrm{H} 5)\right]$ (cell 1) & 2 & 3.38 & 95 & 36 & 0.116 \\
\hline$\left[\mathrm{Ru}(\mathrm{bpy})_{2}(\mathrm{H} 5)\right]$ (cell 2) & 2 & 3.34 & 95 & 34 & 0.109 \\
\hline
\end{tabular}




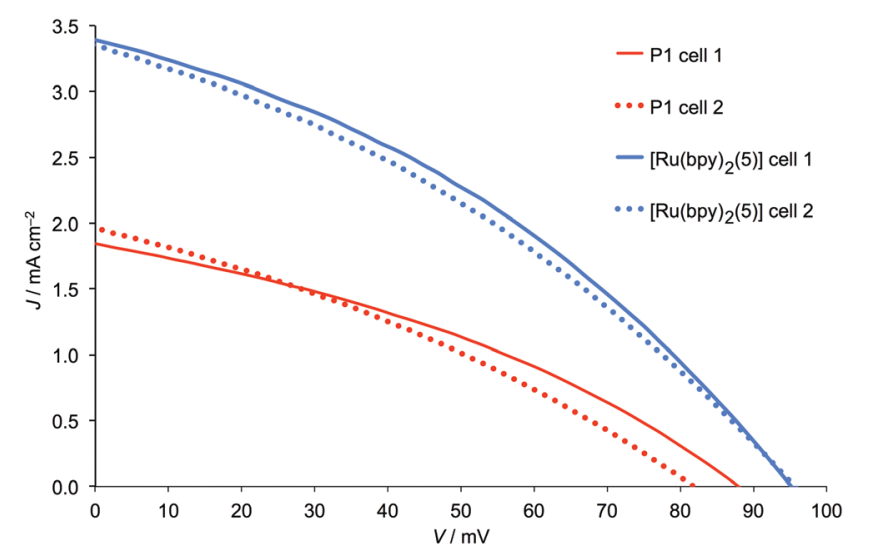

Fig. $9 \mathrm{~J}-\mathrm{V}$ curves for duplicate DSCs containing dyes $\mathrm{P} 1$ and $\left[\mathrm{Ru}(\mathrm{bpy})_{2}(\mathrm{H} 5)\right]$.

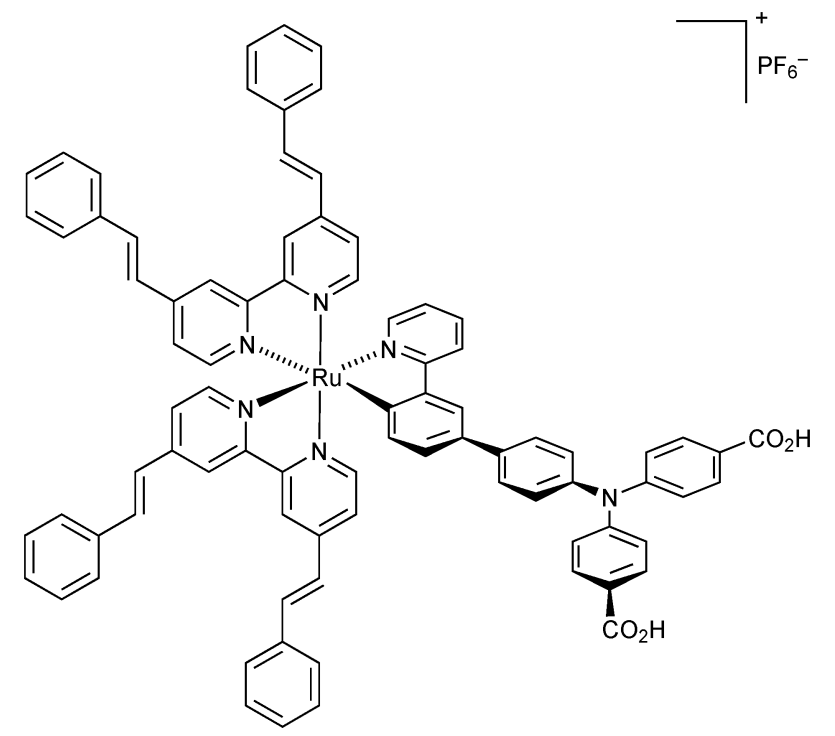

Scheme 7 Structure of a cyclometallated ruthenium dye that achieves $J_{\mathrm{SC}}=3.43 \mathrm{~mA} \mathrm{~cm}^{-2}$ and $\eta=0.104 \%{ }^{9}$

dyes $\left(J_{\mathrm{SC}}=1.50\right.$ and $2.25 \mathrm{~mA} \mathrm{~cm}^{-2}, \eta=0.038$ and $\left.0.079 \%\right) .{ }^{49}$ Values of $V_{\mathrm{OC}}=93 \mathrm{mV}$ and $\mathrm{ff}=33 \%$ for the dye in Scheme 7 compare favourably with the observed values of $V_{\mathrm{OC}}$ and ff (Table 3) for $\left[\mathrm{Ru}(\mathrm{bpy})_{2}(\mathrm{H} 5)\right]$. Given that $\left[\mathrm{Ru}(\mathrm{bpy})_{2}(\mathrm{H} 5)\right]$ is a model dye, its promising performance suggests that the phosphonic anchor is beneficial. Furthermore, while DFT calculations indicate that the anchoring unit is most beneficially attached to the cyclometallating ring which contributes significantly to the HOMO of a $\left[\mathrm{Ru}\left(\mathrm{N}^{\wedge} \mathrm{N}\right)_{2}\left(\mathrm{C}^{\wedge} \mathrm{N}\right)\right]^{+}$complex, ${ }^{11}\left[\mathrm{Ru}(\mathrm{bpy})_{2}(\mathrm{H} 5)\right]$ achieves a value of $J_{\mathrm{SC}}=3.38 \mathrm{~mA} \mathrm{~cm}{ }^{-2}$ with the anchoring unit in the 4-position of the pyridine ring of the $\mathrm{C}^{\wedge} \mathrm{N}$ ligand.

\section{Conclusions}

We have described a readily adaptable modular strategy for cyclometallated ruthenium(II) complexes $\left[\mathrm{Ru}(\mathrm{bpy})_{2}(\mathrm{H} 6)\right]\left[\mathrm{PF}_{6}\right]$ and $\left[\mathrm{Ru}(\mathrm{bpy})_{2}(\mathrm{H} 5)\right]$ which possess a carboxylic or phosphonic acid group attached via a phenylene spacer to the 4-position of the pyridine ring in the cyclometalling ligand. The isolation of the zwitterion $\left[\mathrm{Ru}(\mathrm{bpy})_{2}(\mathrm{H} 5)\right]$ versus the cationic $\left[\mathrm{Ru}(\mathrm{bpy})_{2}(\mathrm{H} 6)\right]^{+}$ is consistent with the difference between the $\mathrm{p} K_{\mathrm{a}}$ values of $\mathrm{RPO}_{3} \mathrm{H}_{2}$ and $\mathrm{RCO}_{2} \mathrm{H}$. The key intermediate in the synthetic pathway is the chloro-derivative $\left[\mathrm{Ru}(\mathrm{bpy})_{2}(\mathbf{1})\right]^{+}$which has been structurally characterized as the $\left[\mathrm{PF}_{6}\right]^{-}$salt.

$\left[\mathrm{Ru}(\mathrm{bpy})_{2}(\mathrm{H} 5)\right]$ has been evaluated as a dye in p-type DSCs and its performance compared to that of the standard dye P1; DSC parameters for the latter were first validated against the benchmarking work of Gibson and coworkers. ${ }^{33}$ Duplicate DSCs containing $\left[\mathrm{Ru}(\mathrm{bpy})_{2}(\mathrm{H} 5)\right]$ exhibit values of $J_{\mathrm{SC}}=3.34$ and $3.38 \mathrm{~mA} \mathrm{~cm}^{-2}$ and $\eta=0.116$ and $0.109 \%$ making this structurally simple dye comparable to the best-performing cyclometallated ruthenium(II) dye in p-type DSCs previously reported. ${ }^{11}$ $\left[\mathrm{Ru}(\mathrm{bpy})_{2}(\mathrm{H} 5)\right]$ achieves values of $V_{\mathrm{OC}}=95 \mathrm{mV}$ and $\mathrm{ff}=34-36 \%$. The performance parameters confirm the effectiveness of a phosphonic acid anchor in the dye and the attachment of the anchoring unit to the pyridine ring of the cyclometallating ligand. We are currently investigating the improvement of the performance of this and related ruthenium(II) dyes in p-type DSCs, and are striving to understand the factors that contribute to the surprisingly good performance of the structurally simple $\left[\mathrm{Ru}(\mathrm{bpy})_{2}(\mathrm{H} 5)\right]$.

\section{Acknowledgements}

We thank the Swiss National Science Foundation (Grant number 200020_144500) and the University of Basel for financial support. Chantelle Ekanem and Agron Ilazi are acknowledged for their contributions to optimization of conditions for the preparation of FTO/NiO electrodes. We thank Professor Ernst Meyer for helpful discussions. FIB images were recorded in the Nano Imaging Lab (Swiss Nanoscience Institute, University of Basel) by Daniel Mathys.

\section{References}

1 K. C. D. Robson, P. G. Bomben and C. P. Berlinguette, Dalton Trans., 2012, 41, 7814, and references therein.

2 P. G. Bomben, K. C. D. Robson, B. D. Koivisto and C. P. Berlinguette, Coord. Chem. Rev., 2012, 256, 1438, and references therein.

3 A. Colombo, C. Dragonetti, A. Valore, C. Coluccini, N. Manfredi and A. Abbotto, Polyhedron, 2014, 82, 50, and references therein.

4 T. Bessho, E. Yoneda, J.-H. Yum, M. Guglielmi, I. Tavernelli, H. Imai, U. Rothlisberger, Md. K. Nazeeruddin and M. Grätzel, J. Am. Chem. Soc., 2009, 131, 5930.

5 T. Funaki, H. Otsuka, N. Onozawa-Komatsuzaki, K. Kasuga, K. Sayama and H. Sugihara, J. Mater. Chem. A, 2014, 2, 15945.

6 See for example: J.-Y. Li, C. Lee, C.-Y. Chen, W.-L. Lee, R. Ma and C.-G. Wu, Inorg. Chem., 2015, 54, 10483; J.-F. Huang, J.-M. Liu, P.-Y. Su, Y.-F. Chen, Y. Shen, L.-M. Xiao, D.-B. Kuang and C.-Y. Su, Electrochim. Acta, 2015, 174, 494.

7 P. G. Bomben, K. C. D. Robson, P. A. Sedach and C. P. Berlinguette, Inorg. Chem., 2009, 48, 9631. 
8 J. He, H. Lindström, A. Hagfeldt and S.-E. Lindquist, Sol. Energy Mater. Sol. Cells, 2000, 62, 265.

9 M. He, Z. Ji, Z. Huang and Y. Wu, J. Phys. Chem. C, 2014, 118, 16518.

10 Z. Ji, G. Natu and Y. Wu, ACS Appl. Mater. Interfaces, 2013, 5,8641 .

11 Z. Ji, G. Natu, Z. Huang, O. Kokhan, X. Zhang and Y. Wu, J. Phys. Chem. C, 2012, 116, 16854.

12 Z. Ji and Y. Wu, J. Phys. Chem. C, 2013, 117, 18315.

13 C. J. Wood, K. C. D. Robson, P. I. P. Elliott, C. P. Berlinguette and E. A. Gibson, RSC Adv., 2014, 4, 5782.

14 Y. Pellegrin, L. Le Pleux, E. Blart, A. Renaud, B. Chavillon, N. Szuwarski, M. Boujtita, L. Cario, S. Jobic, D. Jacquemin and F. Odobel, J. Photochem. Photobiol., A, 2011, 219, 235.

15 M. Braumüller, M. Schulz, M. Staniszewska, D. Sorsche, M. Wunderlin, J. Popp, J. Guthmuller, B. Dietzek and S. Rau, Dalton Trans., 2016, 45, 9216.

16 C. D. Ertl, D. P. Ris, S. C. Meier, E. C. Constable, C. E. Housecroft, M. Neuburger and J. A. Zampese, Dalton Trans., 2015, 44, 1557.

17 H. Andersson, F. Almqvist and R. Olsson, Org. Lett., 2007, 9, 1335.

18 C. Edder and J. M. J. Fréchet, Org. Lett., 2003, 5, 1879.

19 Y. Kuninobu, H. Ida, M. Nishi and M. Kanai, Nat. Chem., 2015, 7, 712.

20 Bruker Analytical X-ray Systems, Inc., 2006, APEX2, version 2 User Manual, M86-E01078, Madison, WI.

21 L. Palatinus and G. Chapuis, J. Appl. Crystallogr., 2007, 40, 786.

22 P. W. Betteridge, J. R. Carruthers, R. I. Cooper, K. Prout and D. J. Watkin, J. Appl. Crystallogr., 2003, 36, 1487.

23 I. J. Bruno, J. C. Cole, P. R. Edgington, M. K. Kessler, C. F. Macrae, P. McCabe, J. Pearson and R. Taylor, Acta Crystallogr., Sect. B: Struct. Sci., 2002, 58, 389.

24 C. F. Macrae, I. J. Bruno, J. A. Chisholm, P. R. Edgington, P. McCabe, E. Pidcock, L. Rodriguez-Monge, R. Taylor, J. van de Streek and P. A. Wood, J. Appl. Crystallogr., 2008, 41, 466.

25 L. Zhang and J. M. Cole, ACS Appl. Mater. Interfaces, 2015, 7, 3427.

26 E. C. Constable and J. M. Holmes, J. Organomet. Chem., 1986, 301, 203.

27 W. Runguphan and S. E. O'Connor, Org. Lett., 2013, 15, 2850.

28 See for example: J. Moon and S. Lee, J. Organomet. Chem., 2009, 694, 473.

29 Metal Phosphonate Chemistry: From Synthesis to Applications, ed. A. Clearfield and K. Demadis, RSC Publishing, 2011, ch. 5 , p. 166.

30 P. Reveco, W. R. Cherry, J. Medley, A. Garber, R. J. Gale and J. Selbin, Inorg. Chem., 1986, 25, 1842.

31 M. L. Muro-Small, J. E. Yarnell, C. E. McCusker and F. N. Castellano, Eur. J. Inorg. Chem., 2012, 4004.
32 D. Dini, Y. Halpin, J. G. Vos and E. A. Gibson, Coord. Chem. Rev., 2015, 304-305, 179.

33 C. J. Wood, G. H. Summers, C. A. Clark, N. Kaeffer, M. Braeutigam, L. R. Carbone, L. D’Amario, K. Fan, Y. Farré, S. Narbey, F. Oswald, L. A. Stevens, C. D. J. Parmenter, M. W. Fay, A. La Torre, C. E. Snape, B. Dietzek, D. Dini, L. Hammarström, Y. Pellegrin, F. Odobel, L. Sun, V. Artero and E. A. Gibson, Phys. Chem. Chem. Phys., 2016, 18, 10727.

34 Unpublished results: T. Kosmalski, Master Thesis, University of Basel, 2015.

35 X. L. Zhang, F. Huang, A. Nattestad, K. Wang, D. Fu, A. Mishra, P. Bäuerle, U. Bach and Y.-B. Cheng, Chem. Commun., 2011, 47, 4808.

36 I. R. Perera, T. Daeneke, S. Makuta, Z. Yu, Y. Tachibana, A. Mishra, P. Bäuerle, C. A. Ohlin, U. Bac and L. Spiccia, Angew. Chem., Int. Ed., 2015, 54, 3758.

37 See for example: S. Powar, T. Daeneke, M. T. Ma, D. Fu, N. W. Duffy, G. Götz, M. Weidelener, A. Mishra, P. Bäuerle, L. Spiccia and U. Bach, Angew. Chem., Int. Ed., 2013, 52, 602; S. Sheehan, G. Naponiello, F. Odobel, D. P. Dowling, A. Di Carlo and D. Dini, J. Solid State Electrochem., 2015, 19, 975; V. Novelli, M. Awais, D. P. Dowling and D. Dini, J. Anal. Chem., 2015, 6, 176.

38 F. Odobel and Y. Pellegrin, J. Phys. Chem. Lett., 2013, 4, 2551.

39 F. Odobel, Y. Pellegrin, F. B. Anne and D. Jacquemin in High-Efficiency Solar Cells: Physics, Materials, and Devices, ed.

X. Wang and Z. M. Wang, Springer, Switzerland, 2014, ch. 8.

40 S. Karamshuk, S. Caramori, N. Manfredi, M. Salamone, R. Ruffo, S. Carli, C. A. Bignozzi and A. Abbotto, Energies, 2016, 9, 33.

41 P. Qin, H. Zhu, T. Edvinsson, G. Boschloo, A. Hagfeldt and L. Sun, J. Am. Chem. Soc., 2008, 130, 8570.

42 P. Qin, M. Linder, T. Brinck, G. Boschloo, A. Hagfeldt and L. Sun, Adv. Mater., 2009, 21, 2993.

43 L. Li, E. A. Gibson, P. Qin, G. Boschloo, M. Gorlov, A. Hagfeldt and L. Sun, Adv. Mater., 2010, 22, 1795.

44 L. Zhu, H. Yang, C. Zhong and C. M. Li, Chem. - Asian J., 2012, 7, 2791.

45 P. Qin, J. Wiberg, E. A. Gibson, M. Linder, L. Li, T. Brinck, A. Hagfeldt, B. Albinsson and L. Sun, J. Phys. Chem. C, 2010, 114, 4738.

46 Y.-S. Yen, W.-T. Chen, C.-Y. Hsu, H.-H. Chou, J. T. Lin and M.-C. P. Yeh, Org. Lett., 2011, 13, 4930.

47 F. J. Malzner, S. Y. Brauchli, E. Schönhofer, E. C. Constable and C. E. Housecroft, Polyhedron, 2014, 82, 116.

48 Z. Huang, G. Natu, Z. Ji, M. He, M. Yu and Y. Wu, J. Phys. Chem. C, 2012, 116, 26239.

49 S. Lyu, Y. Farré, L. Ducasse, Y. Pellegrin, T. Toupance, C. Olivier and F. Odobel, RSC Adv., 2016, 6, 19928. 